\title{
Aberrant AZIN2 and polyamine metabolism precipitates tau neuropathology
}

\author{
Leslie A. Sandusky-Beltran,, Andrii Kovalenko, ${ }^{1,2}$ Devon S. Placides, ${ }^{1,2}$ Kevin Ratnasamy, ${ }^{1,2}$ Chao Ma, , ${ }^{1,5,6}$ Jerry B. Hunt Jr., ${ }^{6,7}$ \\ Huimin Liang, ${ }^{6,7}$ John Ivan T. Calahatian,, ${ }^{1,2}$ Camilla Michalski, ${ }^{3}$ Margaret Fahnestock, ${ }^{3}$ Laura J. Blair, ${ }^{1,4}$ April L. Darling, ${ }^{1,4}$ \\ Jeremy D. Baker, ${ }^{1,4}$ Sarah N. Fontaine, ${ }^{1,4}$ Chad A. Dickey, ${ }^{1,4}$ Joshua J. Gamsby, ${ }^{1,4}$ Kevin R. Nash, ${ }^{5}$ Erin Abner, ${ }^{6,8}$ \\ Maj-Linda B. Selenica, ${ }^{6,9}$ and Daniel C. Lee ${ }^{2,6,7}$ \\ 1Byrd Alzheimer's Institute and 'Department of Pharmaceutical Sciences, University of South Florida, Tampa, Florida, USA. ${ }^{3}$ Department of Psychiatry \& Behavioral Neurosciences, McMaster University, \\ Hamilton, Ontario, Canada. ${ }^{4}$ Department of Molecular Medicine and ${ }^{5}$ Department of Molecular Pharmacology and Physiology, University of South Florida, Tampa, Florida, USA. ${ }^{6}$ Sanders-Brown Center on \\ Aging, ${ }^{7}$ Department of Neuroscience, ${ }^{8}$ Department of Epidemiology, and ${ }^{9}$ Department of Molecular \& Cellular Biochemistry, University of Kentucky, Lexington, Kentucky, USA
}

\begin{abstract}
Tauopathies display a spectrum of phenotypes from cognitive to affective behavioral impairments; however, mechanisms promoting tau pathology and how tau elicits behavioral impairment remain unclear. We report a unique interaction between polyamine metabolism, behavioral impairment, and tau fate. Polyamines are ubiquitous aliphatic molecules that support neuronal function, axonal integrity, and cognitive processing. Transient increases in polyamine metabolism hallmark the cell's response to various insults, known as the polyamine stress response (PSR). Dysregulation of gene transcripts associated with polyamine metabolism in Alzheimer's disease (AD) brains were observed, and we found that ornithine decarboxylase antizyme inhibitor 2 (AZIN2) increased to the greatest extent. We showed that sustained AZIN2 overexpression elicited a maladaptive PSR in mice with underlying tauopathy (MAPT P301S; PS19). AZIN2 also increased acetylpolyamines, augmented tau deposition, and promoted cognitive and affective behavioral impairments. Higher-order polyamines displaced microtubule-associated tau to facilitate polymerization but also decreased tau seeding and oligomerization. Conversely, acetylpolyamines promoted tau seeding and oligomers. These data suggest that tauopathies launch an altered enzymatic signature that endorses a feed-forward cycle of disease progression. Taken together, the tau-induced PSR affects behavior and disease continuance, but may also position the polyamine pathway as a potential entry point for plausible targets and treatments of tauopathy, including AD.
\end{abstract}

\section{Introduction}

In the brain, the microtubule-associated protein tau promotes microtubule assembly and the axonal outgrowth of neurons; however, in certain diseases, tau becomes hyperphosphorylated, aggregates, and leads to neuronal death. Tau neuropathology is a hallmark of several neurodegenerative diseases known as tauopathies (1), including Alzheimer's disease (AD). Although pathways underlying the development of tau neuropathology remain unclear, the role of polyamines in neurological disorders, including $\operatorname{AD}(2,3)$, has emerged. Polyamine imbalance may exacerbate disease progression by contributing to tau neuropathology and cognitive impairment.

Polyamines provide an assortment of physiological effects that provide neuronal function, axonal integrity, and cognitive processing (4-7). Under homeostatic conditions, the polyamine pathway remains highly self-regulating. Ornithine decarboxylase

Authorship note: $C A D$ is deceased.

Conflict of interest: The authors have declared that no conflict of interest exists. Copyright: () 2021, American Society for Clinical Investigation. Submitted: November 21, 2018; Accepted: December 16, 2020; Published: February 15, 2021.

Reference information: J Clin Invest. 2021;131(4):e126299.

https://doi.org/10.1172/JCl126299.
(ODC) remains the rate-limiting step in the biosynthesis of polyamines, as it is responsible for converting ornithine to putrescine and subsequent synthesis to spermidine and spermine. ODC activity, and more specifically translation, varies in response to cellular perturbation, most notably polyamine concentration (8). ODC degradation depends on a polyamine-activated antizyme (AZ, also referred to as an "antizyme for ODC"). AZ inhibits ODC with high affinity and prevents activity-dependent dimerization (9). Furthermore, AZ can be sequestered and inhibited by antizyme inhibitors (AZINs) (See Figure 1A for simplified pathway), which associates with the positive regulation of ODC activity and polyamine biosynthesis.

Self-regulation of the pathway also occurs by polyamine retroor back-conversion. Higher-order polyamines recycle back to their lower-order precursors via the catabolic enzymes spermine oxidase (SMOX), spermidine/spermine- $N^{1}$-acetyltransferase (SSAT), and polyamine oxidase (PAOX). The back-conversion produces acetylated metabolites of the polyamines, which either recycle to lower-order polyamines or persist as putatively inactive forms; however, the functional roles of acetylpolyamines remain unclear.

Increasing reports indicate that specific stimuli, either emotional or physical, can elicit a polyamine stress response (PSR), resulting in altered central polyamine homeostasis (10). Although adaptive elevations in polyamines after a short-term stressor 
A Polyamine pathway

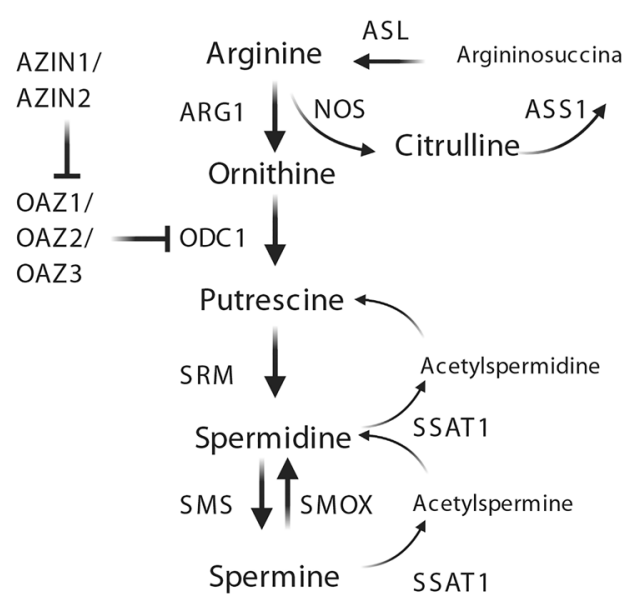

B

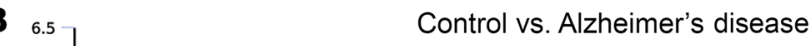

C

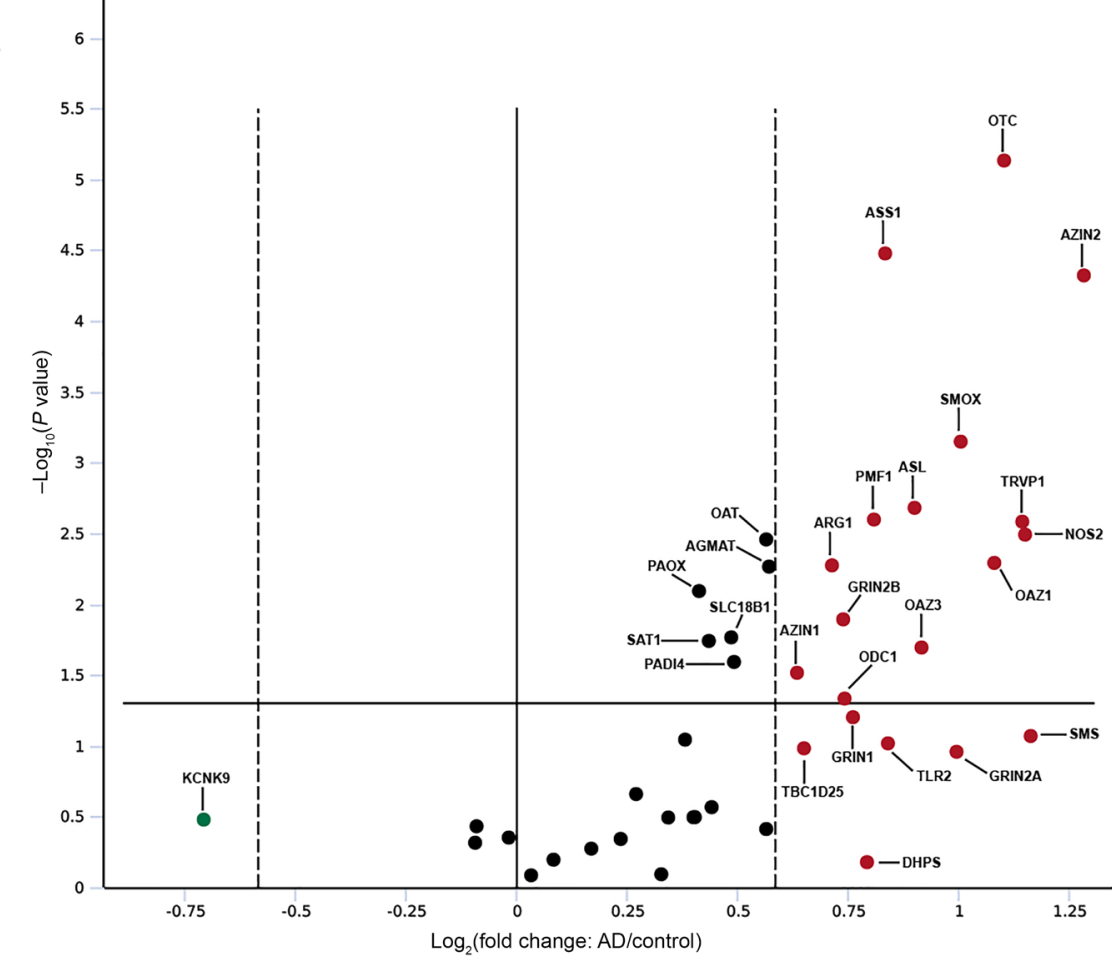

- Upregulated • Unchanged • Downregulated

c Control (C) Alzheimer's disease (AD)

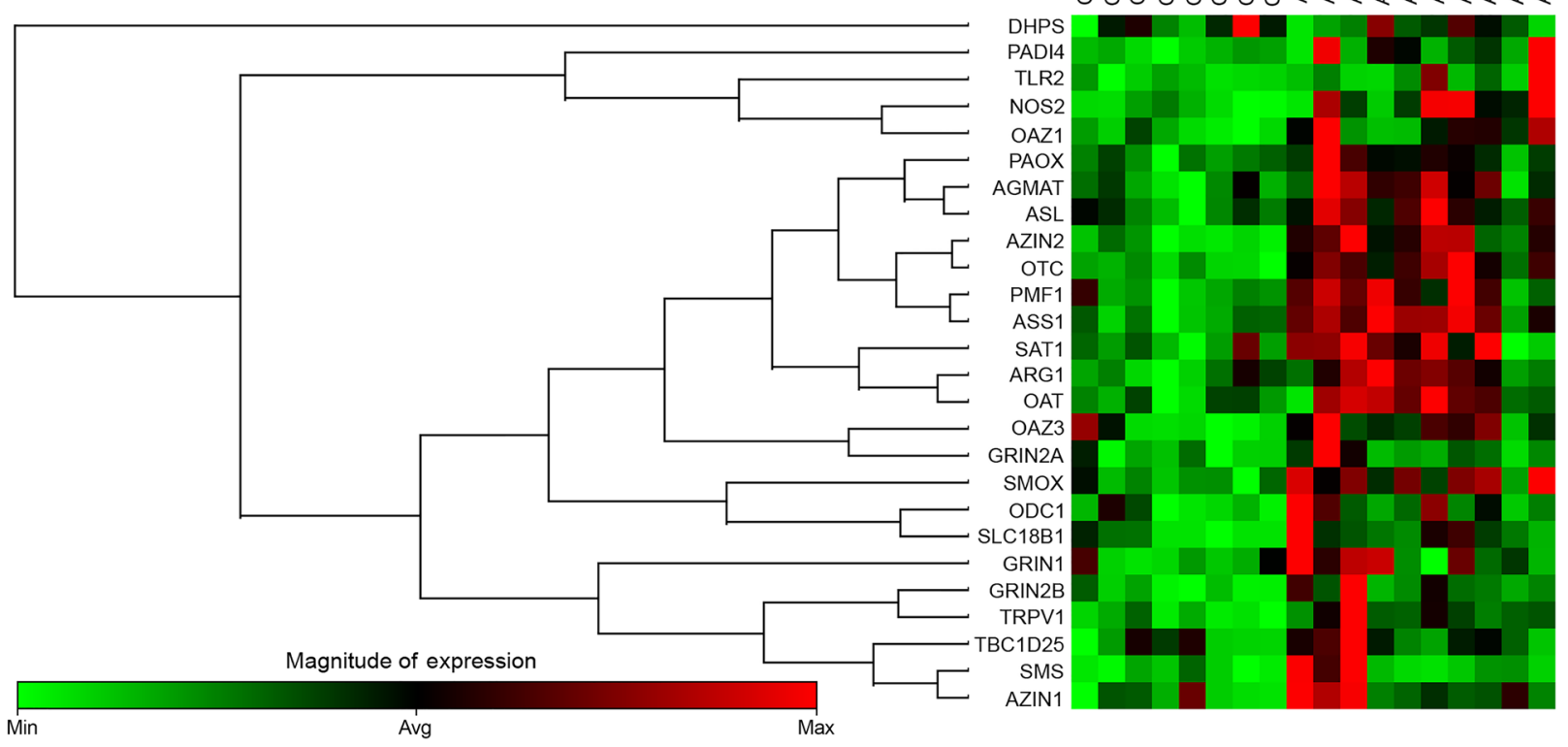

Figure 1. Polyamine transcripts increase in Alzheimer's disease brain. (A) Simplified schematic of the polyamine pathway. Arginase 1 (ARG1), nitric oxide synthase (NOS), arginosuccinate synthetase (ASS1), arginosuccinate lyase (ASL), antizyme inhibitor 2 (AZIN2), ornithine decarboxylase antizyme 1 (OAZ1), ornithine decarboxylase (ODC), spermidine/spermine $N^{1}$-acetyltransferase 1 (SSAT), spermidine synthase (SRM), spermine synthase (SMS), spermine oxidase (SMOX), polyamine oxidase (PAOX). (B and C) Alzheimer's disease brains show significantly dysregulated polyamine-associated gene transcripts. (B) Volcano plot displays statistical significance versus fold change on the $y$ and $x$ axes, respectively. Combining a $P$ value statistical test with the fold regulation enables identification of transcripts with large and small expression changes that are statistically significant. Fold regulation threshold $=1.5, P$ value threshold $=0.05$. (C) Clustergram with nonsupervised hierarchical clustering of significantly dysregulated gene transcripts to display a heat map with dendrograms indicating coregulated genes. Data was obtained using RT Profiler PCR array gene expression data analysis software from Qiagen. 


\section{Table 1. Gene transcripts: Alzheimer's disease versus control}

\begin{tabular}{lccc} 
Gene name & Cene symbol & Fold change & $\boldsymbol{P}$ value \\
Antizyme inhibitor 2 & AZIN2 & 2.43 & 0.0000 \\
Spermine synthase & SMS & 2.24 & 0.0851 \\
Nitric oxide synthase 2 & NOS2 & 2.22 & 0.0032 \\
Transient receptor potential cation channel subfamily V member 1 & TRPV1 & 2.21 & 0.0026 \\
Ornithine carbamoyltransferase & OTC & 2.15 & 0.0000 \\
Ornithine decarboxylase antizyme1 & OAZ1 & 2.12 & 0.0051 \\
Spermine oxidase & SMOX & 2.01 & 0.0007 \\
Clutamate ionotropic receptor NMDA type subunit 2A & CRIN2A & 2.00 & 0.1099 \\
Ornithine decarboxylase antizyme3 & OAZ3 & 1.89 & 0.0202 \\
Argininosuccinate lyase & ASL & 1.87 & 0.0021 \\
Toll-like receptor 2 & TLR2 & 1.79 & 0.0959 \\
Argininosuccinate synthase 1 & ASS1 & 1.78 & 0.0000 \\
Polyamine-modulated factor 1 & PMF1 & 1.75 & 0.0025 \\
Deoxyhypusine synthase & DHPS & 1.73 & 0.6620 \\
Clutamate ionotropic receptor NMDA type subunit 1 & CRIN1 & 1.70 & 0.0627 \\
Ornithine decarboxylase 1 & ODC1 & 1.67 & 0.0463 \\
Clutamate ionotropic receptor NMDA type subunit 2B & CRIN2B & 1.67 & 0.0128 \\
Arginase 1 & ARG1 & 1.64 & 0.0053 \\
TBC1 domain family member 25 & TBC1D25 & 1.57 & 0.1039 \\
Antizyme inhibitor 1 & AZIN1 & 1.55 & 0.0305 \\
Agmatinase & ACMAT & 1.49 & 0.0054 \\
Ornithine aminotransferase & OAT & 1.48 & 0.0035 \\
Peptidyl arginine deiminase 4 & PADI4 & 1.41 & 0.0255 \\
Solute carrier family 18 member B1 & SLC18B1 & 1.40 & 0.0171 \\
Spermidine/spermine N1-acetyltransferase 1 & SAT1 & 1.35 & 0.0181 \\
Polyamine oxidase & PAOX & 1.33 & 0.0081 \\
\hline
\end{tabular}

Gene transcripts reflect Figure 1. $P<0.05$ and fold change $>1.5$. hippocampal tissue from $\mathrm{AD}$ brains compared with control brains (Figure 1, B and C, and Table 1). Transcripts reflecting altered arginine metabolism included increased ASS1; ASL1, which promotes arginine synthesis and NOS2; and ARG1, which depletes arginine to produce nitric oxide and ornithine. Transcripts associated with polyamine signaling included increased prosynthetic enzymes $S M S$ and $O D C 1$ and catabolic SAT1, SMOX, and PAOX enzymes, signifying dysregulation along the entire cycle of the polyamine axis. $P M F 1$ also increased, which associates with increase SAT1 transcription together with NRF2 (16). Positive indirect regulators of polyamine production consisted of increased AZIN1, AZIN2, and AGMAT, whereas negative regulators consisted of $O A Z 1, O A Z 3, O A T 1$, and OTC. Interestingly, $\mathrm{AD}$ brains showed elevated vesicular polyamine transporter SLC18B1 (17). Amongst the NMDA receptors, only the glutamate ionotropic receptor NMDA type subunit 2B (GRIN2B) increased. Most notably, of all the gene transcripts, the greatest change was AZIN2 (fold change $=2.4341, P=0.000048$ ) .

AZIN2 protein is increased in AD brains. We measured protein in the human postmortem $\mathrm{AD}$ brain for tau and AZIN2 levels to extend our transcriptome findings. Using a cluster analysis based on paired helical filament (PHF) phospho-tau (AT8) in AD, we observed significant increases in high molecular weight (HMW) tau, phospho-tau (AT8, PHF1), and AZIN2 in

might serve as beneficial, persistent stress and long-term PSR activation can become maladaptive, resulting in chronic polyamine dysregulation. Altered regulation in this pathway can occur at the level of the prosynthetic and catabolic enzymes, as well as their respective polyamine and acetylated products $(2,3,11-15)$.

In the current study, we found that the gene transcript for AZIN2 was significantly altered in the AD brain compared with control brains. We confirmed increased AZIN2 protein in AD brains associated with phospho-tau. We also found that AZIN2 overexpression promoted a maladaptive PSR in mice with underlying tau neuropathology, higher acetylpolyamines, and increased tau levels, and it precipitated cognitive and affective behavioral impairments. Importantly, nontransgenic (nTg) littermates behaviorally habituated to sustained polyamine activation. These data further cement the notion that the PSR differentially affects tauopathies. Increased polyamine metabolites may serve as mediators of tau neuropathology and potential biomarkers associated with tau, including AD.

\section{Results}

AD brains indicate altered polyamine-associated gene transcripts. To determine how $\mathrm{AD}$ affects the polyamine pathway, we developed a focused PCR array platform harboring gene transcripts associated with arginine metabolism and polyamine signaling (Figure 1A). Polyamine transcripts were significantly changed in postmortem
$\mathrm{AD}$ brains that were positive for AT8 staining (AT8+; Figure 2, $\mathrm{A}-\mathrm{F})$. These data further support the correlation between tau neuropathology and dysregulation of polyamine metabolism regarding elevated AZIN2.

AZIN2 overexpression precipitates tau-dependent alterations in anxiety and cognition. To determine how chronic polyamine activation (sustained PSR) impacted affect and cognition during tau neuropathology, we overexpressed AZIN2 via adeno-associated viral constructs (AAV9-AZIN2) or an empty capsid plasmid in the cortex and hippocampus of nTg and tau PS19 mice (Figure 3A). Immunohistochemistry confirmed equal exogenous AZIN2 HA-tagged viral expression and AZIN2 in the cortex and hippocampus of nTg and PS19 mice, ensuring similar expression across groups (Figure 3, B and C). Although tau (HT7) remained unchanged after increased AZIN2 (Figure 3D), phospho-tau (AT8) increased in the cortex, hippocampus (CA3), and dentate gyrus (DG) in response to AZIN2 overexpression in PS19 mice (Figure 3E). PS19 mice showed an increase in microglia activation in the hippocampus's DG relative to $\mathrm{nTg}$ controls (Figure 3F) and independent of AZIN2. These data suggest that chronic polyamine activation may facilitate tau neuropathology.

AZIN2 overexpression induced tau-dependent alterations in affective and cognitive processing (Figure 4, A-F). Overall, locomotor activity was comparable across all groups in terms of the total distance traveled (Figure 4A); however, AZIN2 overexpres- 
A
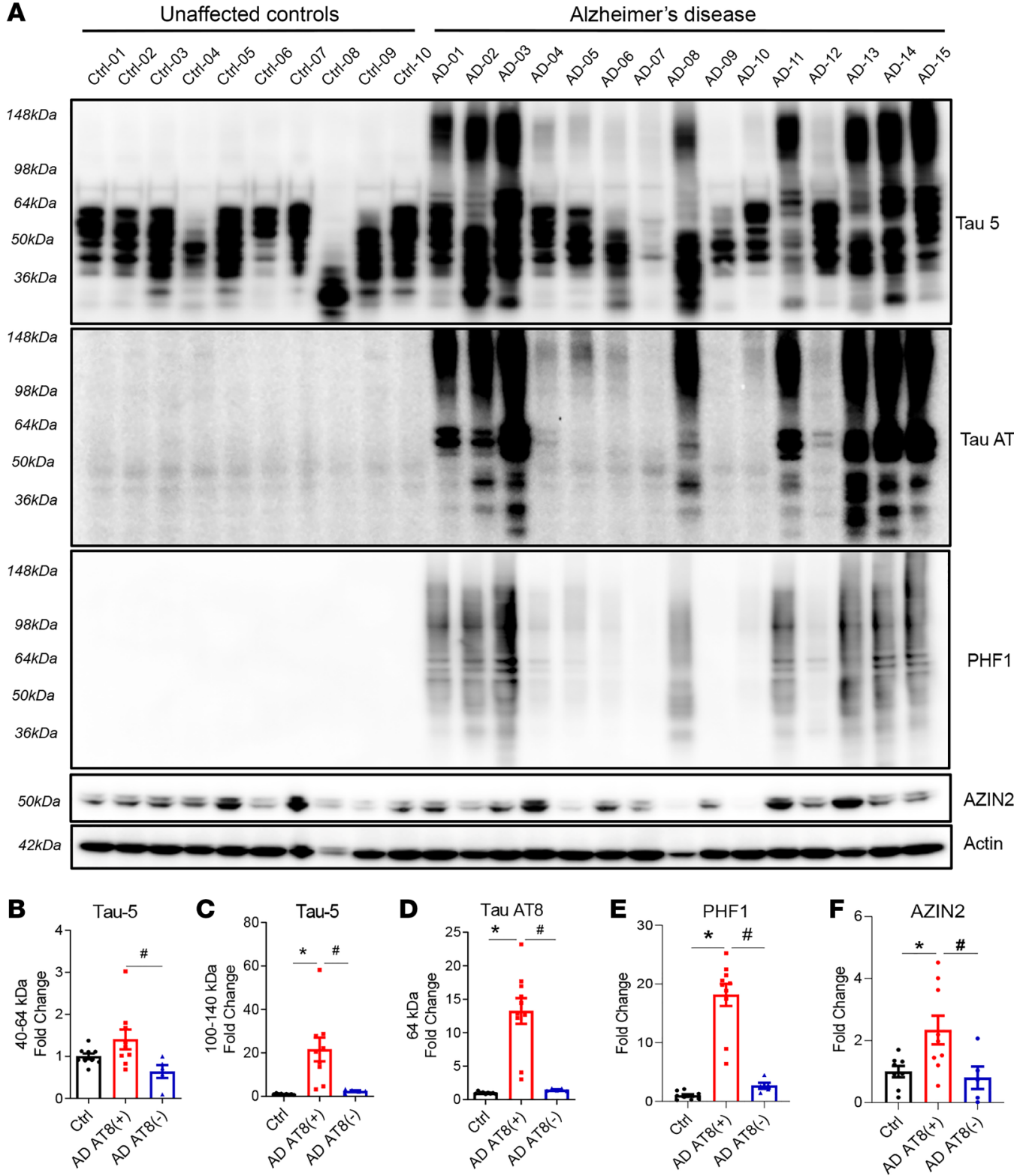

Figure 2. AZIN2 is increased in Alzheimer's disease brains. (A) Images and quantification of Western blot analysis of human cortex for tau (Tau-5, AT8) and AZIN2. (B-F) A 1-way ANOVA revealed a group difference (control, AD positive for AT8 [AD/AT8+]) and AD negative for AT8 (AD/AT8-) on total tau (Tau5, 40-64 kDa), HMW total tau (Tau-5, 100-140 kDa), tau AT8, PHF1, and AZIN2 ( $P=0.032 ; P=0.001 ; P=0.000 ; P=0.000 ; P=0.044$, respectively). The $\left(\mathrm{AD} / \mathrm{AT}^{+}\right)$group showed increased Tau-5 relative to the $\left(\mathrm{AD} / \mathrm{AT}^{-}\right)$) group $(P=0.029)$, an increase in HMW Tau-5 relative to the control group $(P=0.001)$ and the (AD/AT8 $)$ group $(P=0.009)$, an increase in AT8 relative to the controls $(P=0.000)$ and the (AD/AT8 $)$ group $(P=0.000)$, and an increase in PHF1 relative to the control group $(P=0.000)$ and the $\left(\mathrm{AD} / \mathrm{AT} 8^{-}\right)$group $(P=0.000)$. AZIN2 increased in the $\left(\mathrm{AD} / \mathrm{AT}^{+}\right)$group relative to the control group $(P=$ $0.0366)$ and the (AD/AT8 $)$ group $(P=0.0365)$. One-way ANOVA followed by post hoc comparisons using Tukey's HSD, $n=5-9$, data represented by scatter plot with bar. ${ }^{*} P<0.05$; control versus $\left(\mathrm{AD} / \mathrm{AT}^{+}\right) ;{ }^{\#} P<0.05$; $\left(\mathrm{AD} / \mathrm{AT}^{+}\right)$versus (AD/AT8 ${ }^{-}$).

sion increased anxiety in tau transgenic mice, as measured by decreased entries to the center zone and decreased time in the center zone (Figure 4, B and C). As determined by percentage of alternation in the $\mathrm{Y}$ maze, working memory declined in tau mice with chronic PSR activation (Figure 4D). Finally, inhibition and fear-associated memory recall, measured by latency to cross in the inhibitory avoidance task, was impaired in mice with tauopathy and chronic AZIN2 overexpression (Figure 4, E and F). These data indicate that $\mathrm{nTg}$ littermates adapted behaviorally to chronic polyamine activation; however, underlying tauopathy promoted a unique maladaptive behavioral phenotype that impacted affect and cognitive performance in response to chronic PSR activation.

Existing tauopathy augments the PSR after chronic AZIN2 overexpression. To determine how the polyamine system regulates itself in response to sustained polyamine activation by AZIN2 overexpression and tau neuropathology, we quantified brain putrescine, spermidine, spermine, and acetylspermidine levels (Figure 5, A-D). Overall, there was no change in spermidine 
A
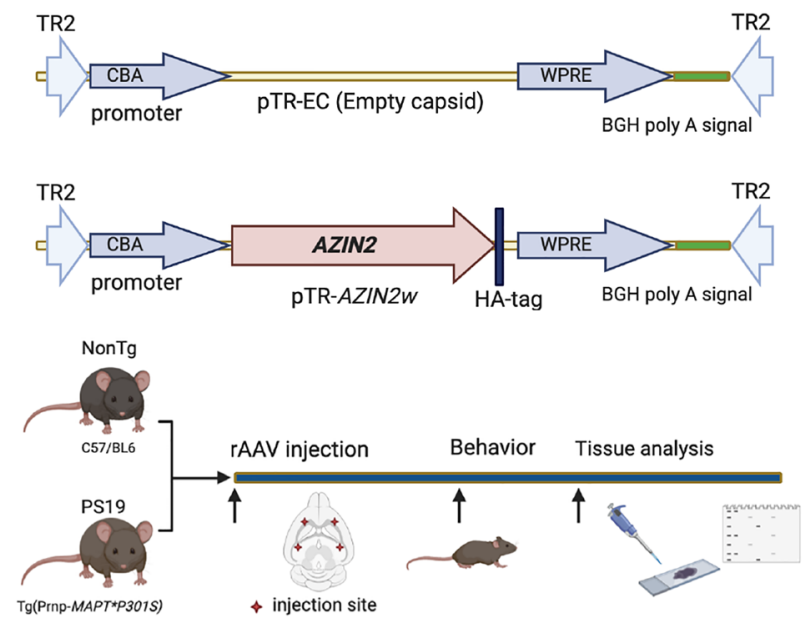

C
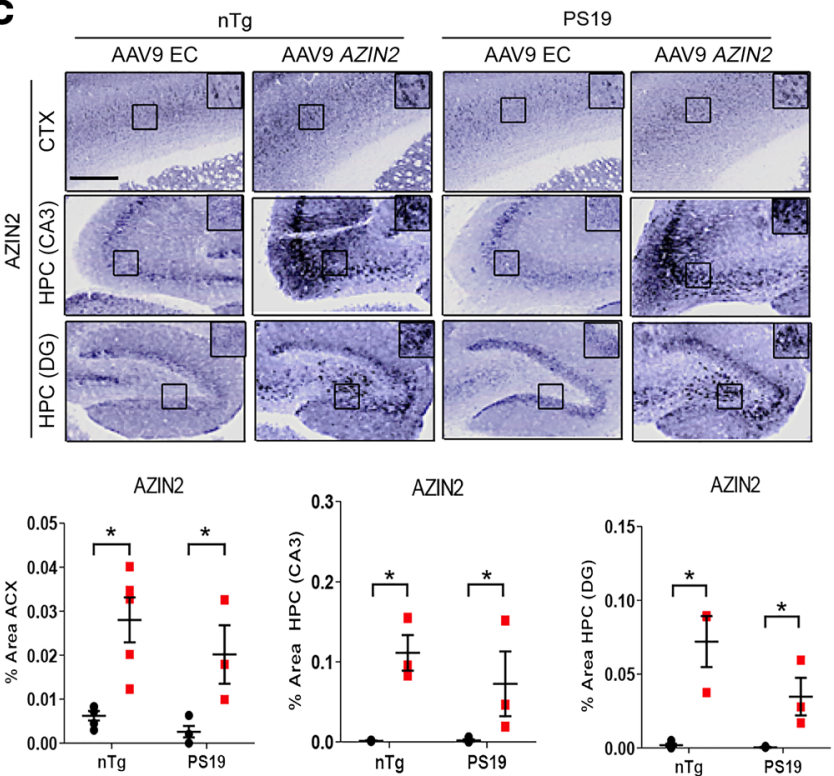

$\mathbf{E}$
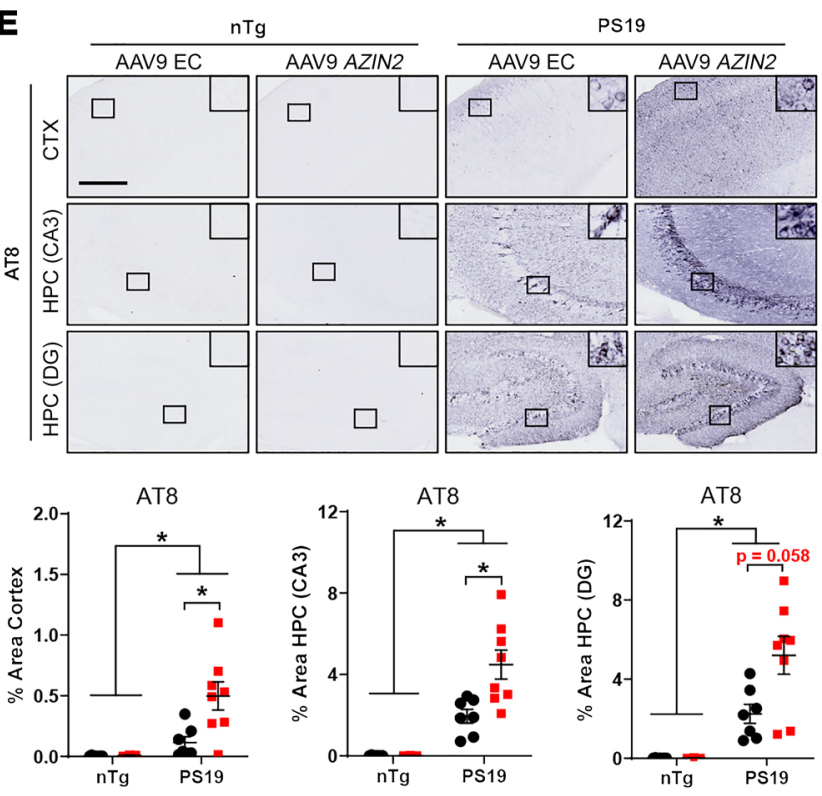

B
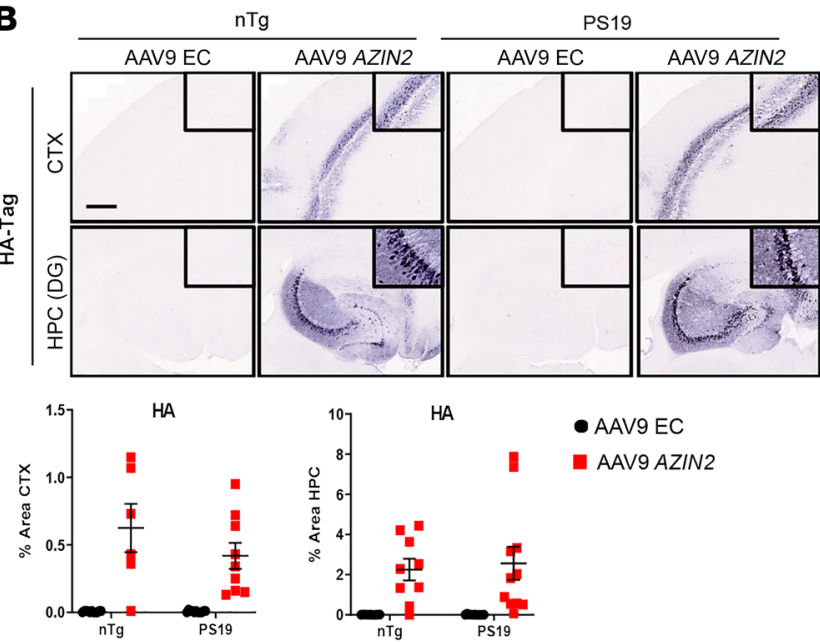

D
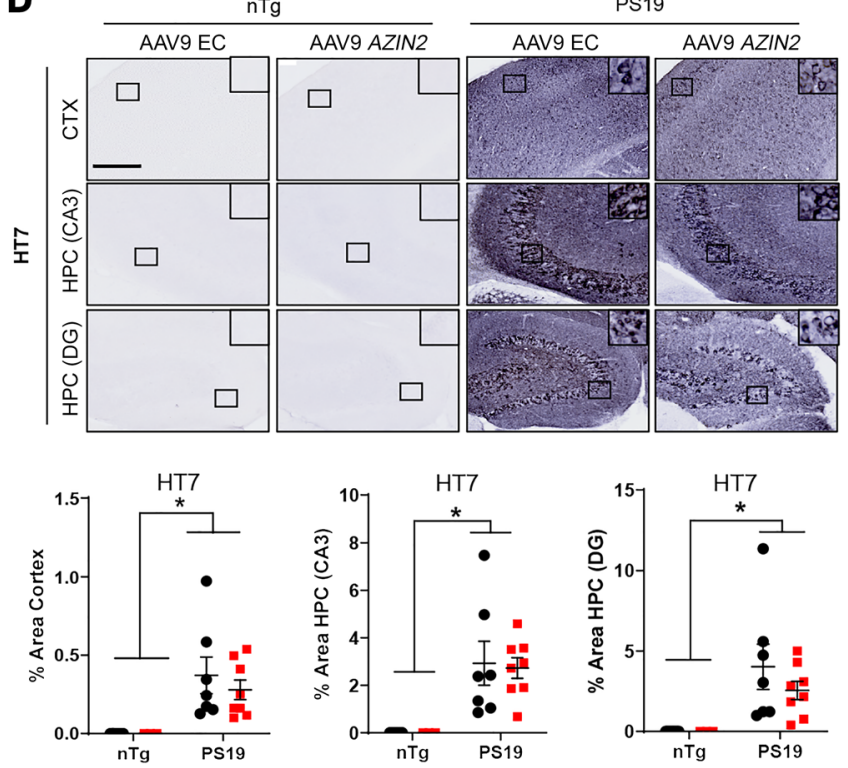

$\mathbf{F}$

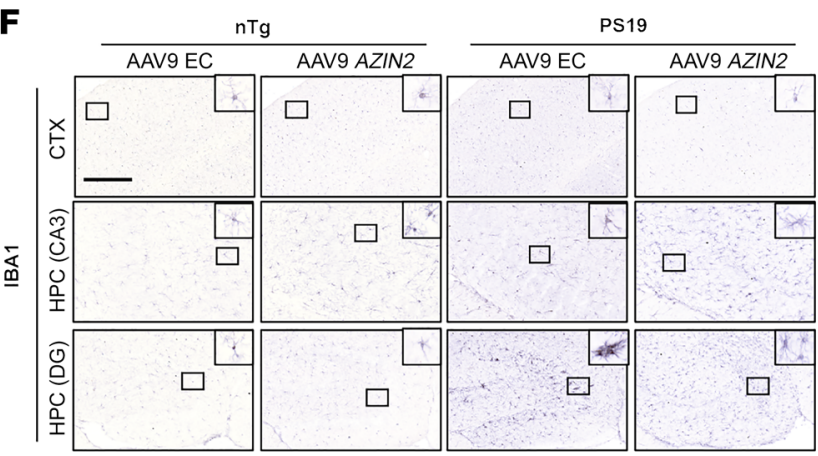

IBA1
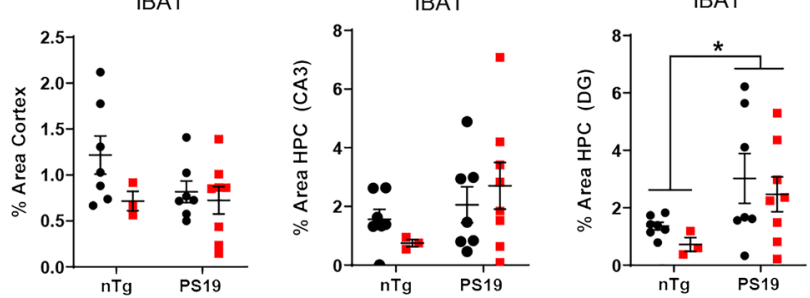
Figure 3. Overexpression of AZIN2 increased regional phosphorylated tau in the mouse CNS. (A-F) AZIN2 overexpression in a mouse model of tauopathy. (A) AAV9-AZIN2 viral construct, cortical and hippocampal injection sites, respective genotypes, and outcome measures used in the present study. (B) Cortical and hippocampal AAV9-AZIN2 expression by HA staining. The main effects analysis showed no interaction in the cortex ( $P=0.172)$ or hippocampus $(P=0.604)$, ensuring equal expression. (C) There was no effect of genotype on AZIN2 in the dentate gyrus (DC) $(P=$ 0.42 ), but a treatment effect on AZIN2 expression in the ACX, CA3, and DC $(P=0.000, P=0.000, P=0.000$, respectively). (D) There was a main effect of genotype on tau (HT7) in the CTX, CA3, and DC $(P=0.001 ; P=$ $0.000 ; P=0.002$, respectively). (E) There was a main effect of genotype on tau (AT8) in the CTX, CA3, and DC $(P=0.002 ; P=0.000 ; P=0.002$, respectively); treatment in the $C T X, C A 3$, and a trend toward a main effect in the DG $(P=0.036 ; P=0.028 ; P=0.057$, respectively). Lastly, there was an interaction of genotype and treatment on AT8 in the CTX, CA3, and a trend toward an interaction in the DC $(P=0.040 ; P=0.028 ; P=0.058$, respectively). Pairwise comparisons revealed AZIN2 overexpression increased AT8 in the CTX, CA3, and DC in PS19 mice $(P=0.001, P=0.001$, $P=0.003$, respectively). (F) There was a main effect of genotype on IBA1 in the $\mathrm{DG}(P=0.022)$. A $2 \times 2$ factorial ANOVA was performed, followed by pairwise comparisons using Sidak's posttest correction, $n=3-11$, data represented by mean \pm SEM. ${ }^{*} P<0.05$. Scale bar: $500 \mu \mathrm{m}$ for panel B; $\times 20$ original magnification ( $6 \%$ zoom). Scale bar: $50 \mu \mathrm{m}$ for panels $\mathbf{C}-\mathbf{F} ; \times 20$ original magnification ( $15 \%$ zoom = images; $60 \%$ zoom = insets).

levels between groups, possibly because spermidine shows the highest abundance of all polyamines; however, putrescine and acetylspermidine significantly increased in response to AAV-AZIN2 overexpression, and this response was augmented in PS19 mice (Figure 5, A and D), indicating an interaction of polyamine activation and tau neuropathology through the induction of polyamine back-conversion.

At the protein level, exogenous AZIN2 was increased in response to AAV-AZIN2 overexpression, ensuring equal viral expression between $\mathrm{nTg}$ and PS19-treated mice (Figure 6, A and B), supporting the immunohistochemical distribution of AZIN2. A $50-\mathrm{kDa}$ band of AZIN2 also increased in response to AAV-AZIN2 overexpression in PS19 mice (Figure 6C). Although this band would align with endogenous AZIN2, suggesting intrinsic activation, we cannot rule out a proteolytic product of exogenous AZIN2. Polyamine pathway activation typically couples with catabolic enzymes to normalize polyamine levels; however, we found that in PS19 mice with AZIN2 overexpression, anabolic enzymes (i.e., ODC, SRM, SMS) increased, indicating a maladaptive PSR in tauopathies (Figure 6, D, F, and G). Overall, AZIN2 increased SSAT as a treatment; however, pairwise analysis indicated that the effect was driven in nTg mice (Figure 6H), suggesting that SSAT protein increased to normalize polyamines after chronic activation. This effect was reduced in PS19 mice, although acetylspermidine increased to a greater extent. This may suggest that acetylspermidine accumulates more or is not cleared as readily during tauopathies and the PSR. Conversely, SMOX, which converts spermine to spermidine, decreased in PS19 mice with AZIN2 overexpression (Figure 6E), but was unchanged in nTg littermates, and supported the mean elevation in spermine in AZIN2-treated PS19 mice (Figure 5C). PAOX remained unchanged between groups (Figure 6I). These data suggest that mice with tauopathy launched a unique PSR after chronic polyamine activation by AZIN2 overexpression, but that nTg mice functionally reestablished homeostasis of the polyamine pathway.
AZIN2 overexpression augments soluble, insoluble, and oligomeric tau. Next, we examined whether chronic polyamine activation through AZIN2 overexpression affected tau neuropathology (Figure 7, A-K). Overexpression of AAV-AZIN2 appeared to precipitate monomeric and HMW soluble, insoluble, and oligomeric tau neuropathology in PS19 mice. More specifically, AAV-AZIN2 overexpression increased soluble total tau (H150), AT8 (50-64 kDa), and phospho-epitopes pSer396 (HMW), pSer199/202 (HMW), and PHF1 (Figure 7, A-J). AZIN2 overexpression also increased insoluble total tau (H150; 50-64 kDa), AT8 (50-64 kDa), and PHF1 (Figure 8, A-I). Lastly, AZIN2 overexpression increased oligomeric tau (T22; Figure 9, A-C). These data indicate that chronic polyamine activation through AZIN2 promoted soluble, insoluble, and oligomeric tau forms.

Spermine displaces tau from microtubules but facilitates tubulin polymerization and bundling. To determine the relationship between polyamines, acetylpolyamines, microtubule polymerization, and tau, we performed a tubulin polymerization assay (Figure 10, A-F, and Table 2). Only spermine revealed a rapid increase in tubulin polymerization, namely during elongation (growth phase, Figure 10C); however, acetylspermine failed to promote tubulin polymerization (Figure 10F), suggesting differential effects of spermine versus acetylspermine on microtubule dynamics. To confirm these effects in cell culture, we treated stably transfected HeLa cells with tubulin-fused GFP construct with various spermine concentrations. The mean average for tubulin bundling was elevated with $1 \mathrm{mM}$ (not significant) as quantified by GFP fluorescence (Figure 11, A and B). Finally, to understand the dynamic relationship between tubulin, tau, and polyamines, we performed a cosedimentation assay with HeLa cells that stably overexpressed $4 \mathrm{RON}$ human tau $(\mathrm{C} 3 \mathrm{H} / \mathrm{tau})$. We found that although spermine increased tubulin polymerization, it did so at the expense of displacing tau from microtubules (Figure 12, A-E). Accumulation of spermine reduced free tubulin (Figure 12C) and increased polymerized tubulin (Figure 12B). Conversely, increasing concentrations of spermine-displaced tau were associated with polymerized tubulin (heavy pellet) (Figure 12D) while increasing tau in the soluble fraction (Figure 12E). These data signify that polyamines may acutely serve to stabilize neuronal integrity during a PSR; however, chronic elevation of higher-order polyamines may displace tau from microtubules, leading to cytoplasmic tau accumulation.

Polyamines and acetylpolyamines differentially affect tau fibrillization. To determine whether polyamines affected tau fibrillization, we coincubated recombinant 4RON WT tau with polyamines and acetylpolyamines in a solution using the thioflavin $\mathrm{T}$ assay (ThT assay) (Figure 13, A-F, and Table 3). Polyamines reduced tau fibrillization in a dose-dependent manner, as measured by AUC, and with higher-order polyamines (longer chain length) inhibiting fibrillization to a greater extent and with greater potency (Figure $13, \mathrm{~A}-\mathrm{C})$. Both spermidine and spermine appeared capable of completely blocking tau fibrillization in this system as low as $10 \mu \mathrm{M}$, and importantly, this concentration exists well within the overall brain content of polyamines $(18,19)$. Strikingly, acetylpolyamines displayed a completely different profile for tau fibrillization. In general, acetylpolyamines failed to inhibit tau fibrillization, most notably in the lag and growth phase (Figure 13, D-F). Acetylspermidine, but to a greater extent acetylspermine, began to increase 

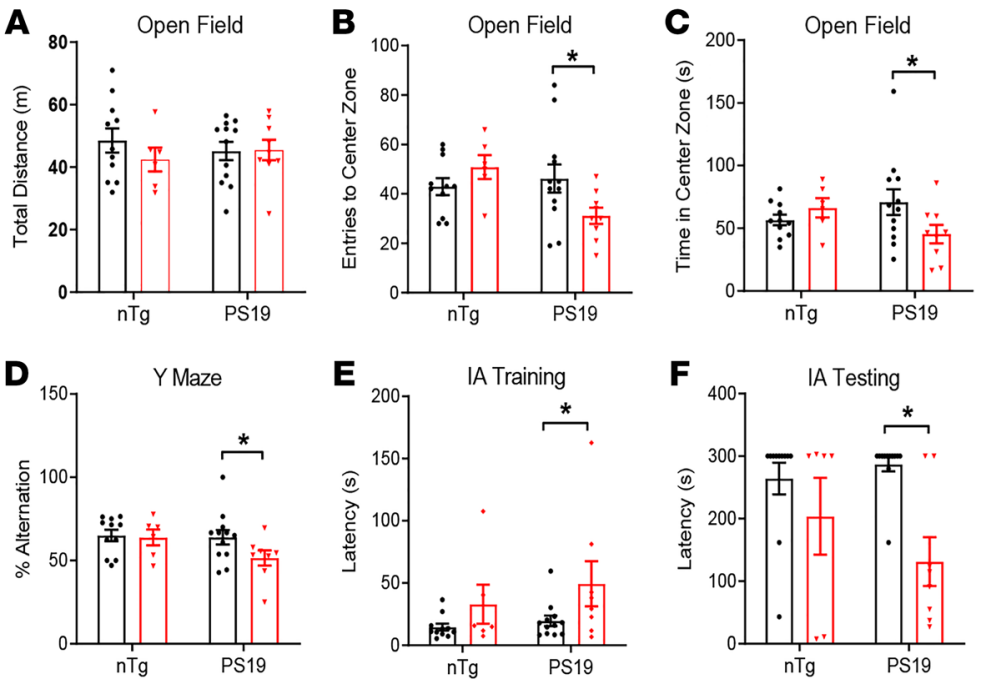

Figure 4. Sustained AZIN2 impacts behavior in tau transgenic mice. (A-F) AZIN2 overexpression precipitates tau-dependent alterations in anxiety and cognition. (A-C) There were no effects of genotype, treatment, or interaction on total distance traveled in the open field; however, a significant interaction was found on the number of entries to the center zone $(P=0.023)$ and time in the center zone $(P=$ 0.044 ), indicating AAV9-AZIN2-precipitated tau-dependent alterations in anxiety. (D) There was no effect of genotype, treatment, or interaction on Y-maze percentage alternation; however, pairwise comparisons revealed a reduction in percentage alternation in PS19 mice $(P=0.046)$, an effect absent in $\mathrm{nTg}$ mice, indicating that AAV9-AZIN2 precipitated a tau-dependent impairment in working memory. (E and F) There was no effect of genotype on latency to cross on day 1 or day 2 ; however, there was a main effect of treatment on latency to cross on day 1 and day $2(P=0.023 ; P=0.002$, respectively). Pairwise comparisons revealed that latency was increased on day 1 and decreased on day 2 in PS19 mice $(P=0.034 ; P=$ 0.001 , respectively), indicating that AAV9-AZIN2 affected tau-dependent basal inhibition and impaired tau-dependent fear-associated memory recall. These effects were not observed in nTg mice. A $2 \times 2$ factorial ANOVA was performed, followed by pairwise comparisons using Sidak's posttest correction, $n=$ 6-12, data represented by mean $\pm \mathrm{SEM} ;{ }^{*} P<0.05$.

tau fibrillization, suggesting that even low $(10 \mu \mathrm{M})$ concentrations of higher-order acetylpolyamines may promote tau fibrillization (Figure 13, E and F).

Spermine prevents higher-order aggregation of tau in $\mathrm{C} 3 \mathrm{H} / \mathrm{tau}$ cells. To further understand polyamines and tau interaction, we used $\mathrm{C} 3 \mathrm{H} /$ tau cells with increasing concentrations of putrescine, spermidine, and spermine for 72 hours (Figure 14, A-M). Sperure $15, \mathrm{~A}-\mathrm{C})$. Cotransfection of both plasmids, GFP10C (1-212 aa)-Tau and GFP11C (213-228 aa)-Tau, in naive N2a cells successfully caused GFP protein complementation and fluoresced green (Figure 15A). Subsequently, we continually selected for a monoclonal N2a-ssGT cell line to stably express the 2 split GFPTau proteins. By coupling the BioTek Cytation 3 Cell Imaging Multi-Mode Reader and Accuri C6 flow cytometer, we were able
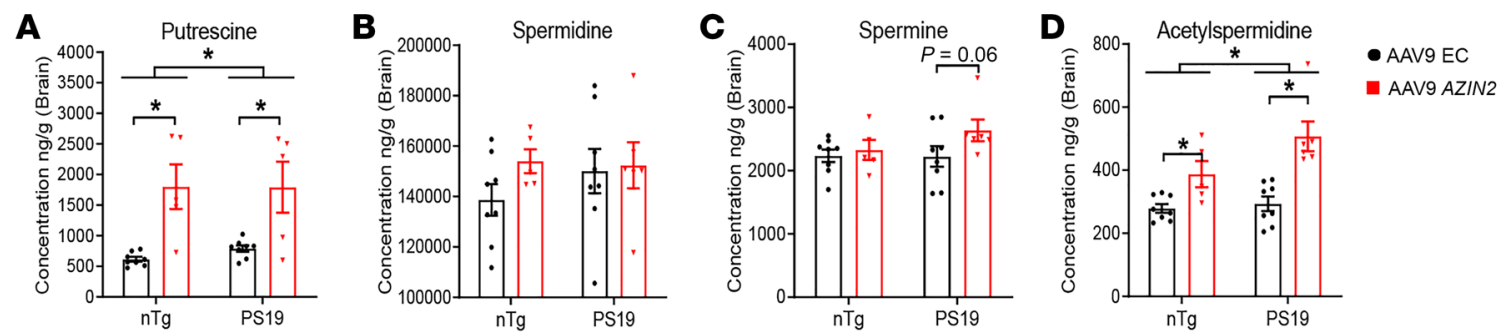

Figure 5. AZIN2 overexpression induces polyamine and acetylpolyamine accumulation. (A) There was an effect of genotype $(P=0.023)$ and treatment $(P=$ 0.000 ) on putrescine; however, pairwise comparisons revealed that while AAV9-AZIN2 increased putrescine in $\mathrm{nTg}(P=0.000)$ and PS19 mice $(P=0.000)$, there was also a significant difference between nTg AAV9-AZIN2-treated mice and PS19 AAV9-AZIN2-treated mice $(P=0.028)$, indicating an interaction between tau and AAV9-AZIN2-induced putrescine. (B) There was no effect of genotype, treatment, or interaction on spermidine. (C) There was no effect of genotype, treatment, or interaction on spermine; however, pairwise comparisons revealed a trend toward increased spermine in AAV9-AZIN2-treated PS19 mice $(P=0.060)$. (D) There was an effect of genotype $(P=0.040)$ and an effect of treatment $(P=0.000)$ on acetylspermidine; however, pairwise comparisons revealed that while AAV9-AZIN2 increased acetylspermidine in $\mathrm{nTg}(P=0.024)$ and PS19 mice $(P=0.000)$, there was also a significant difference between $\mathrm{nTg}$ AAV9-AZIN2-treated mice and PS19 AAV9-AZIN2-treated mice $(P=0.019)$, indicating an interaction between tau and AAV9-AZIN2-induced acetylspermidine. A $2 \times 2$ factorial ANOVA was performed, followed by pairwise comparisons using Sidak's posttest correction, $n=5-8$, data represented by mean $\pm S E M ;{ }^{*} P<0.05$. 
A

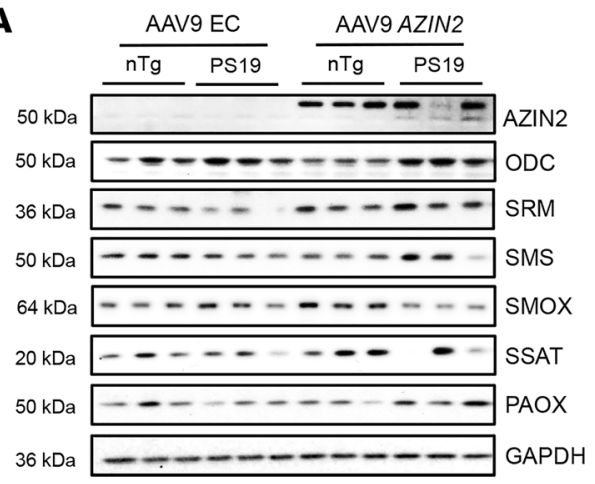

B
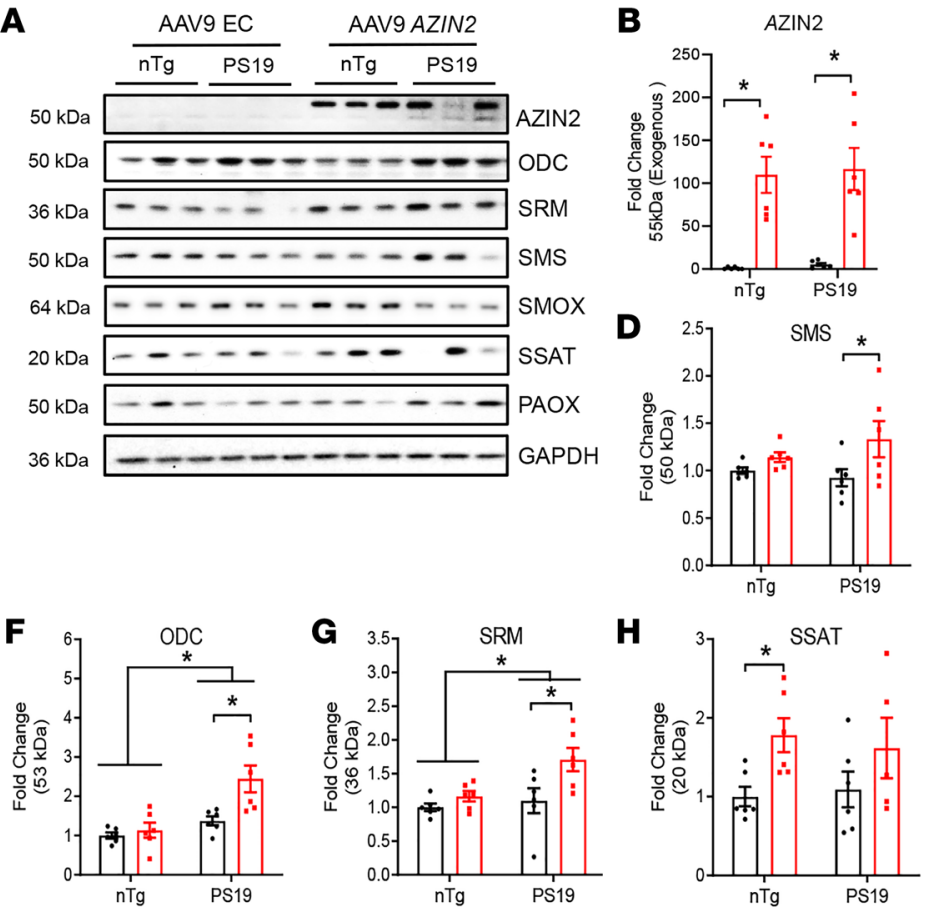
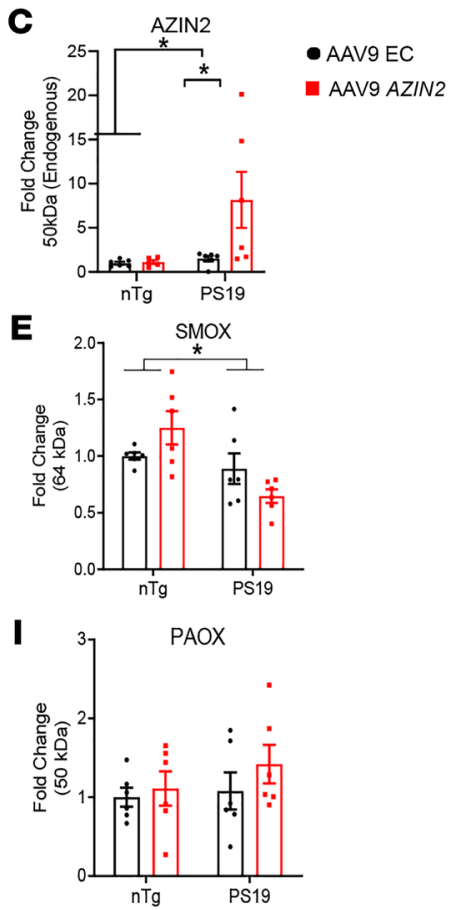

Figure 6. AZIN2 overexpression induces tau-dependent effects on polyamine enzymes. (A) Representative Western blot images of hippocampal polyamine enzyme dysregulation. (B) There was an effect of treatment $(P=0.000)$ on exogenous AZIN2 levels ( $55 \mathrm{kDa})$ but no difference between nTg AAV9-AZIN2-treated and PS19 AAV9-AZIN2-treated mice $(P=0.682)$, indicating equal viral expression across groups. (C) There was an effect of genotype $(P=0.012)$, a trend toward an effect of treatment $(P=0.068)$, and a significant interaction $(P=0.045)$ on endogenous AZIN2 levels (50 kDa). (D) There was an effect of treatment $(P=0.022)$ on SMS. Pairwise comparisons revealed AAV9-AZIN2 increased SMS in PS19 mice $(P=0.017)$. (E) There was an effect of genotype $(P=0.012)$, treatment $(P=0.003)$, and an interaction $(P=0.030)$ on SMOX. (F) There was an effect of genotype $(P=0.001)$, treatment $(P=0.009)$, and interaction of genotype and treatment $(P=0.036)$ on ODC. Pairwise comparisons revealed AAVg-AZIN2 increased ODC in PS19 mice $(P=$ 0.002). (G) There was an effect of genotype $(P=0.027)$ and treatment $(P=0.009)$ on SRM. Pairwise comparisons revealed AAV9-AZIN2 increased SRM in PS19 mice $(P=0.004)$. (H) There was an effect of treatment $(P=0.014)$ on SSAT. Pairwise comparisons revealed AAVg-AZIN2 increased SSAT in nTg mice $(P=0.031)$. (I) No effect of genotype, treatment, or interaction of genotype and treatment detected on PAOX. A $2 \times 2$ factorial ANOVA was performed, followed by pairwise comparisons using Sidak's posttest correction, $n=5-6$, data represented by mean \pm SEM; ${ }^{*} P<0.05$.

to demonstrate both GFP fluorescence images (Figure 15B) and GFP percentage (44.7\%, Figure 13C) in N2a-ssGT cells.

N2a-ssGT cells were incubated with polyamines or acetylpolyamines for 72 hours and tau oligomerization was measured by GFP fluorescence and percentage. Spermidine and spermine decreased oligomerization (Figure 15, D and G-J), and acetylputrescine and acetylspermine increased tau oligomerization (Figure 15, D-F, I, and J). Although it is difficult to predict the various endogenous concentrations of polyamines and acetylpolyamines within this cell line and their impacts on tau biology, these data signify a beneficial role for the accumulation of at least some polyamines in reducing tau oligomerization and a detrimental role for the accumulation of acetylated products on tau oligomerization.

Polyamines and acetylpolyamines differentially affect tau aggregation and seeding. Next, using Tau RD P301S FRET biosensor cells (ATCC, CRL-3275) and human recombinant tau441 (2N4R) P301S mutant preformed fibrils (Tau [P301S] PFFs), we determined how polyamines and acetylpolyamines affect tau aggregation and seeding (Figure 15, A and B). After a 48-hour incubation, FRET cells were treated with 30,100 , or $300 \mu \mathrm{M}$ spermidine, acetylspermidine, spermine, or acetylspermine and $10 \mathrm{nM}$ Tau (P301S) PFFs and were imaged and quantified for percentage fluorescence using flow cytometry. In this model, spermidine ameliorated tau aggregation (Figure 16, C and D) and seeding, whereas acetylspermidine and acetylspermine exacerbated tau aggregation and seeding (Figure 16, C-F), again highlighting the bidirectionality of polyamines and their acetylated byproducts on disease continuance.

\section{Discussion}

Reports show that AZIN2 protein expression occurs along the axons of AD brains. Its expression pattern appears vesicle-like distributed in the somas of selected cortical pyramidal neurons and colocalized with NMDA receptors (20). Although polyamine dysregulation occurs in tauopathies $(2,3,21-23)$, its direct relationship with tau neuropathology is less clear (24). Herein, we confirmed polyamine dysregulation in AD brains and showed that chronic AAV-AZIN2 overexpression increased tau neuropathology in PS19 mice, suggesting that AZIN2 might contribute to disease progression. Polyamines may serve multiple roles depending on the beginning, progression, and end stages of the disease. Recent reports suggest that even peripheral polyamine dysregulation is detectible in $\mathrm{AD}$ and can be used to predict patients with mild cognitive impairment converting to AD up to 2 years earlier than conventional clinical diagnosis (21). These data suggest polyam- 


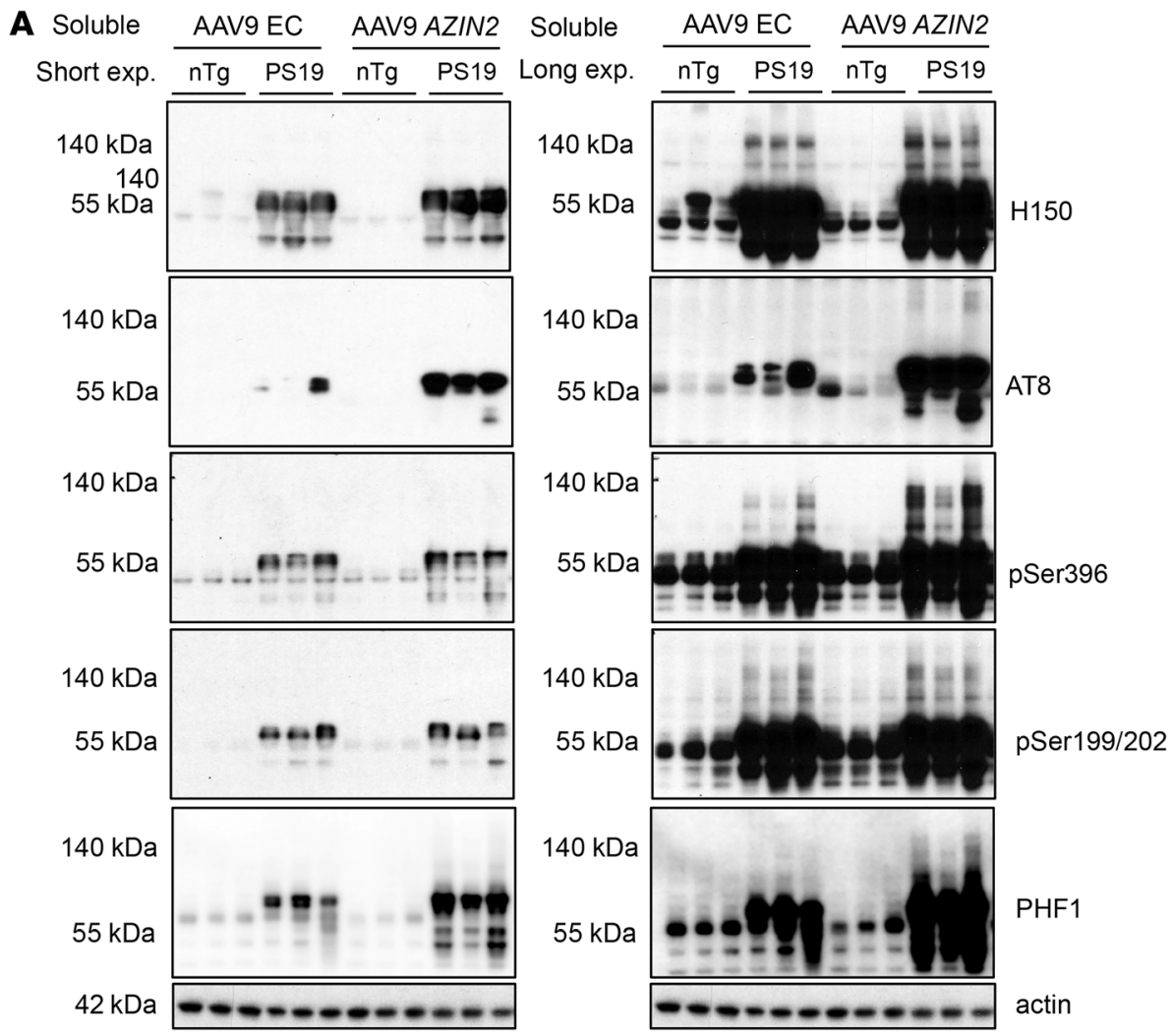

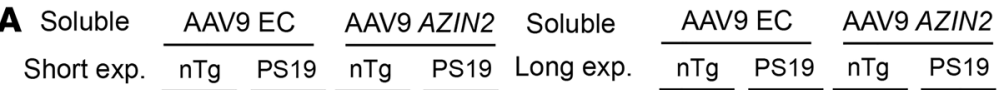

- AAV9 EC

- AAV9 AZIN2
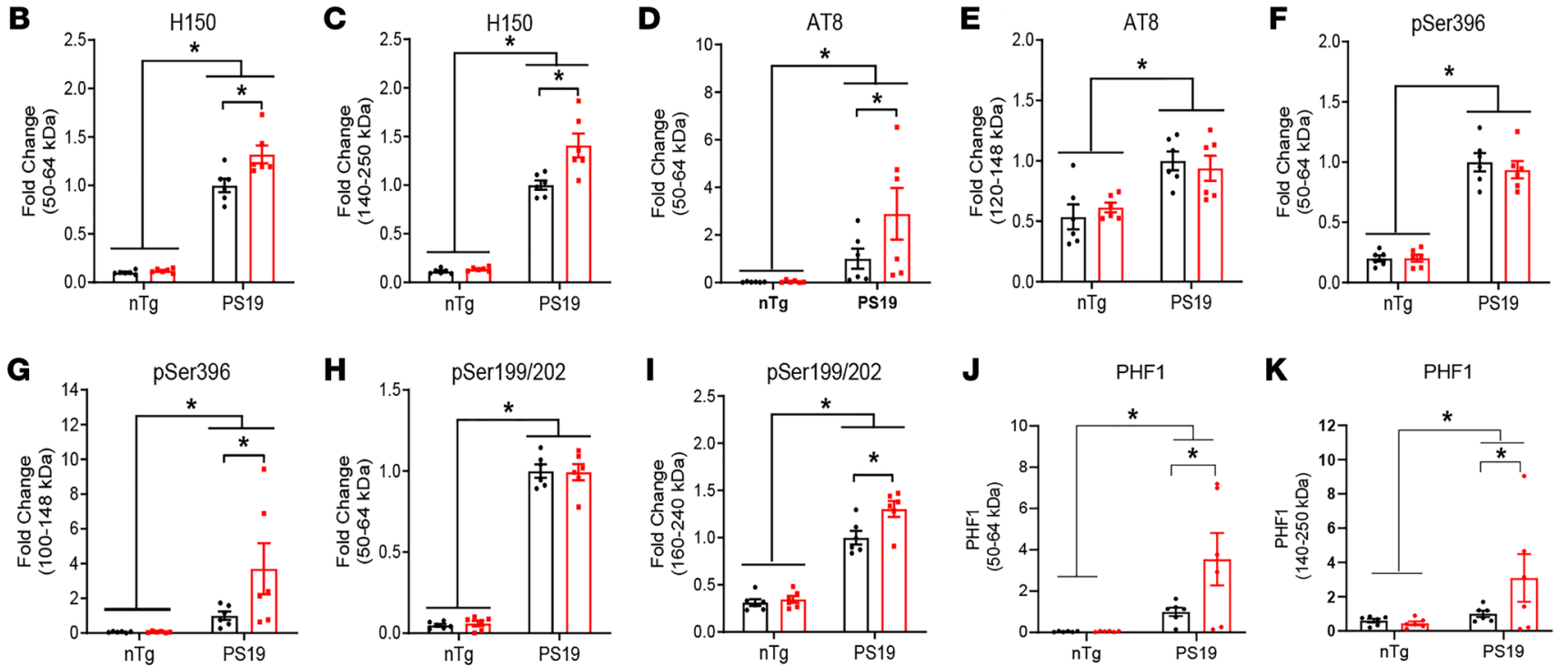

Figure 7. AZIN2 overexpression increases soluble tau species. (A) Representative Western blot images of hippocampal soluble tau. (B) There was an effect of genotype $(P=0.000)$, treatment $(P=0.008)$, and an interaction $(P=0.017)$ on total tau $\mathrm{H} 150(50 \mathrm{kDa})$. (C) Pairwise comparisons revealed AAV9-AZIN2 increased tau H150 (50 kDa) in PS19 mice $(P=0.001)$. There was an effect of genotype $(P=0.000)$, treatment $(P=0.004)$, and an interaction $(P=0.008)$ on HMW H150 (>140 kDa). Pairwise comparisons revealed AAV9-AZIN2 increased HMW tau H150 in PS19 mice $(P=0.000)$. (D) There was an effect of genotype $(P=0.004)$ on AT8 $(50-64 \mathrm{kDa})$. Pairwise comparisons revealed AAV9-AZIN2 increase AT8 in PS19 mice $(P=0.032)$. (E) There was an effect of genotype $(P=0.000)$ on HMW AT8. (F) There was an effect of genotype $(P=0.000)$ on pSer396 $(50-64 \mathrm{kDa})$ and HMW pSer396 $(P=0.006)$. (C) Pairwise comparisons revealed AAV9-AZIN2 increased HMW pSer396 in PS19 mice $(P=0.003)$. (H) There was an effect of genotype $(P=0.000)$ on pSer199/202 (50-64 kDa). (I) There was an effect of genotype $(P=0.000)$, treatment $(P=0.012)$, and an interaction $(P=0.038)$ on HMW pSer199/202. (J) There was an effect of genotype $(P=0.002)$ on monomeric PHF1 (50 kDa). Pairwise comparisons revealed AAV9-AZIN2 increased monomeric PHF1 in PS19 mice $(P=0.011)$. (K) There was an effect of genotype $(P=0.043)$ on HMW PHF1 (140-250 kDa). Pairwise comparisons revealed AAV9-AZIN2 increased HMW PHF1 in PS19 mice $(P=0.048)$. A $2 \times 2$ factorial ANOVA was performed, followed by pairwise comparisons using Sidak's posttest correction, $n=6$, data represented by mean $\pm \mathrm{SEM} ;{ }^{*} P<0.05$. 

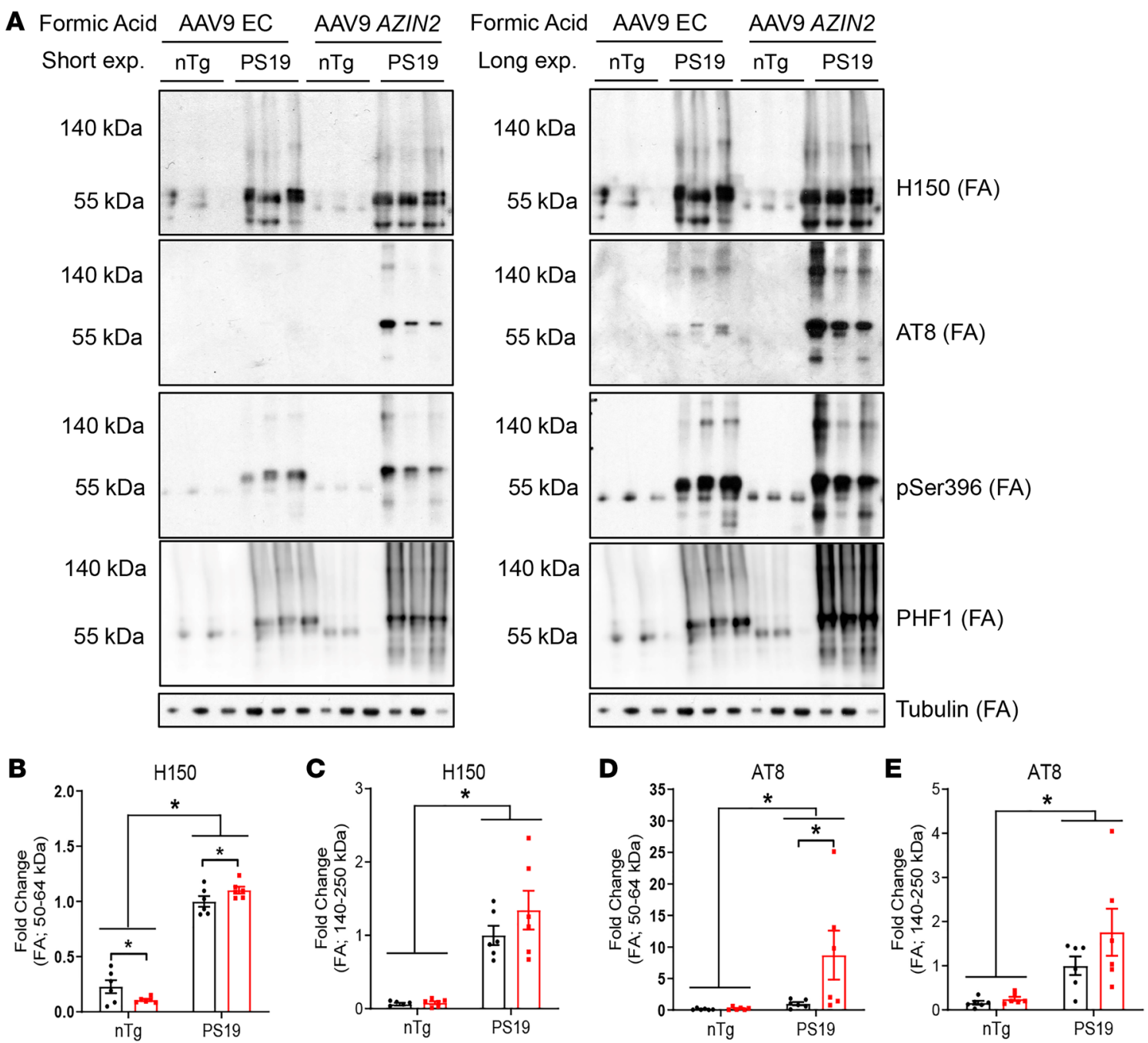

- AAV9 EC
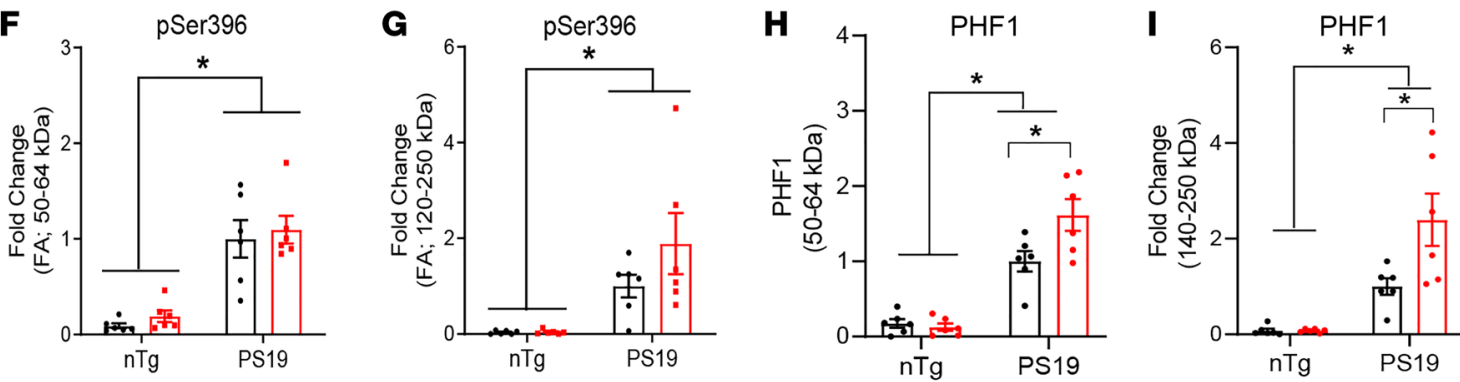

Figure 8. AZIN2 overexpression increases insoluble tau species. (A) Representative Western blot images of hippocampal insoluble tau. (B) There was an effect of genotype $(P=0.000)$ and an interaction $(P=0.014)$ with insoluble total tau (H150) $(50-64 \mathrm{kDa})$. (C) There was an effect of genotype $(P=0.000)$ on insoluble HMW tau H150. (D) There was an effect of genotype $(P=0.026)$ on insoluble AT8 $(50-64 \mathrm{kDa})$. Pairwise comparisons revealed AAV9-AZIN2 increased insoluble AT8 (50-64 kDa) in PS19 mice $(P=0.011)$. (E) There was an effect of genotype $(P=0.001)$ on insoluble HMW AT8. (F) There was an effect of genotype $(P=0.000)$ on insoluble pSer396 (50-64 kDa). (C) There was an effect of genotype $(P=0.001)$ on insoluble HMW pSer396. (H) There was an effect of genotype $(P=0.002)$, treatment $(P=0.043)$, and an interaction $(P=0.020)$ with insoluble PHF1 $(50-64 \mathrm{kDa})$. Pairwise comparisons revealed AAVg-AZIN2 increased PHF1 (50-64 kDa) in PS19 mice $(P=0.003)$. (I) There was an effect of genotype $(P=0.000)$, treatment $(P=0.025)$, and an interaction $(P=0.025)$ with insoluble HMW PHF1 (140-250 kDa). Pairwise comparisons revealed AAV9-AZIN2 increased PHF1 (50-64 kDa) in PS19 mice $(P=0.003)$. A $2 \times 2$ factorial ANOVA was performed, followed by pairwise comparisons using Sidak's posttest correction, $n=6$, data represented by mean $\pm S E M$; ${ }^{*} P<0.05$.

ine system dysfunction and acetylated byproducts as potential biomarkers for tauopathies, including AD.

Behaviorally, a unique interaction between polyamine activation, tau pathology, affect, and cognition emerged during these studies. For example, the increased latency to cross on training day 1 of inhibitory avoidance testing would typically indicate increased basal inhibition and presumably increase latency on day 2; however, this was not the case. We observed a decreased latency to 
A

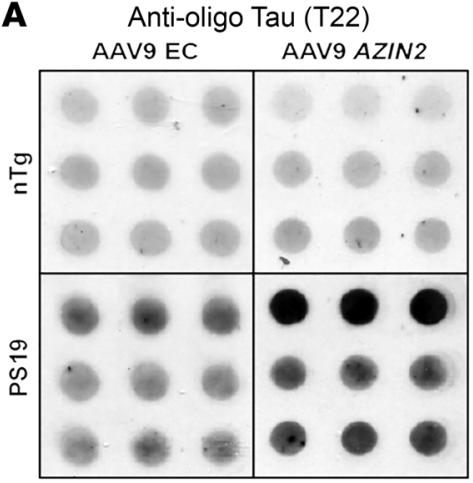

B

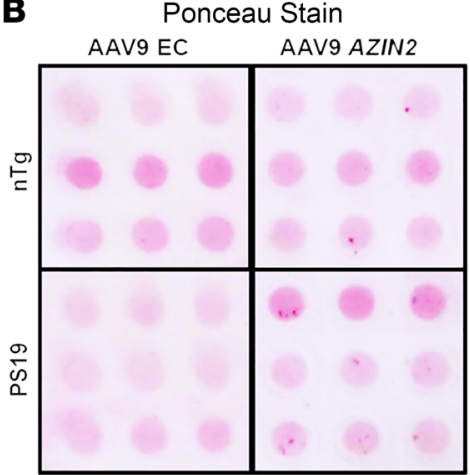

C

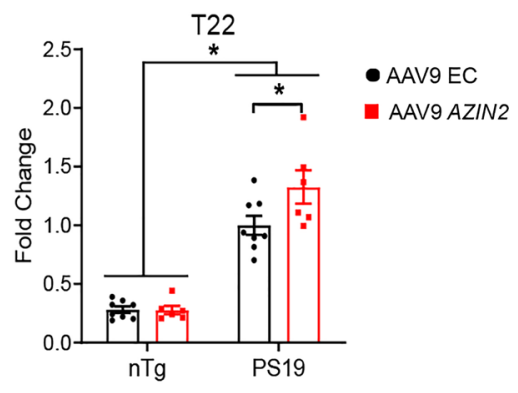

Figure 9. AZIN2 overexpression precipitates oligomeric tau neuropathology. (A) Representative dot blot images of hippocampal oligomeric tau neuropathology. (B) There was an effect of genotype $(P=0.000)$, a trend toward an effect of treatment $(P=0.054)$, and an interaction $(P=0.049)$. Pairwise comparisons revealed AAV9-AZIN2 increased T22 oligomeric tau in PS19 mice $(P=0.008)$. A $2 \times 2$ factorial ANOVA was performed, followed by pairwise comparisons using Sidak's posttest correction, $n=6-8$, data represented by mean $\pm \mathrm{SEM} ;{ }^{*} P<0.05$.

cross on day 2, indicating impaired fear-associated memory recall in PS19 AAV9-AZIN2-treated mice. Although there are numerous reports exploring polyamines and cognitive processing (25), effective regulation (26), and suicidality (27-29), additional measures of AZIN2 overexpression and/or polyamine dysregulation on affective processing should be further explored, particularly in the context of dementias. More clearly, we observed the precipitation of anxiety-related behavior, as seen in the open-field task, and impairment in working memory, as seen in the Y maze, identifying what we believe is a unique impact of tauopathy in response to chronic PSR activation on cognitive and affective processing.

PS19 mice show impairments in spatial learning and memory in the Morris water maze as early as 6 months (30) and impair- ments in contextual fear conditioning as early as 7.5 months (31). However, we did not detect behavioral impairments in 8-monthold PS19 mice treated with AAV9-empty capsid compared with nTg mice treated with AAV9-empty capsid. The phenotypic drift of up to 6 months has been described in PS19 mice and variability in onset and severity of tau pathology (32), which may explain why no significant cognitive impairments were detected in the PS19 AAV9-empty capsid mice. Nonetheless, the lack of behavioral impairment despite underlying tau pathology at this age might mirror cognitive reserve or resilience as seen in some patients, and activation of the PSR during this window precipitated additional tau phenotypes; however, additional research remains necessary in other models of tauopathy.
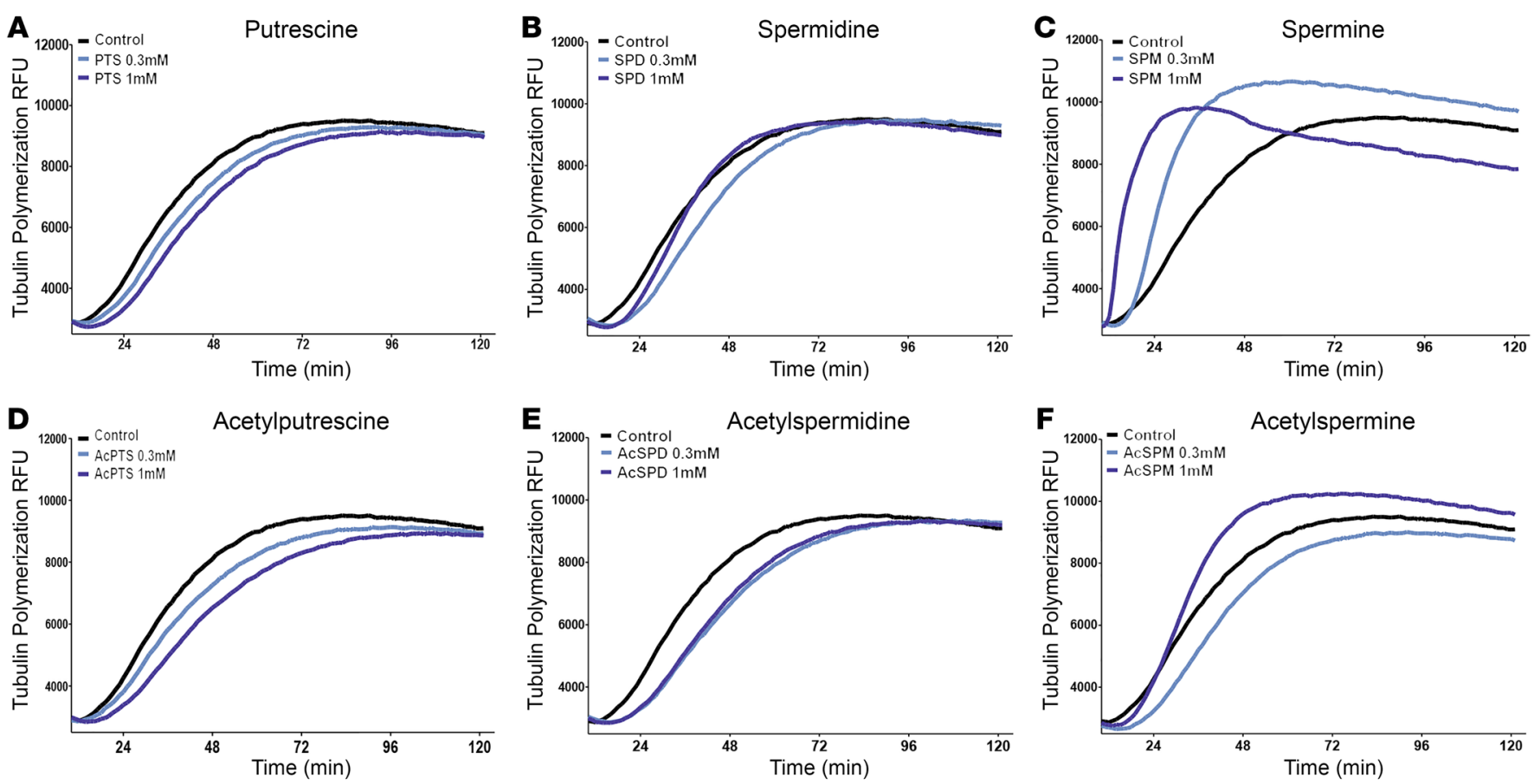

Figure 10. Polyamines and acetylpolyamines differentially affect tubulin polymerization. (A-F) Tubulin polymerization assay and treatment of polyamines (putrescine, A; spermidine, B; spermine, C) or acetylpolyamines (acetylputrescine, D; acetylspermidine, E; acetylspermine, F), followed by quantification of the AUC compared with vehicle controls. Data represented as triplicate averages, and Table 2 reflects AUC change from vehicle control. 


\section{Table 2. Tubulin polymerization}

\begin{tabular}{ccc}
$\begin{array}{c}\text { Putrescine } \\
\text { Control }\end{array}$ & \% Vehicle & $\%$ Change \\
\hline $0.3 \mathrm{mM}$ & 96 & -4 \\
$1 \mathrm{mM}$ & 92 & -8 \\
\hline $\begin{array}{c}\text { Spermidine } \\
\text { Control }\end{array}$ & $\%$ Vehicle & $\%$ Change \\
\hline $0.3 \mathrm{mM}$ & 96 & -4 \\
\hline $1 \mathrm{mM}$ & 99 & -1 \\
\hline Spermine & $\%$ Vehicle & $\%$ Change \\
\hline Control & 116 & 16 \\
\hline $0.3 \mathrm{mM}$ & 108 & 8 \\
\hline $1 \mathrm{mM}$ & $\%$ Vehicle & $\%$ Change \\
\hline Acetylputrescine & & -6 \\
\hline Control & 94 & -11 \\
\hline $0.3 \mathrm{mM}$ & 89 & $\%$ Change \\
\hline $1 \mathrm{mM}$ & $\%$ Vehicle & -8 \\
\hline Acetylspermidine & & -7 \\
\hline Control & 92 & $\%$ Change \\
\hline $0.3 \mathrm{mM}$ & 93 & -9 \\
\hline $1 \mathrm{mM}$ & $\%$ Vehicle & 8 \\
\hline Acetylspermine & & \\
\hline Control & 91 & \\
\hline $0.3 \mathrm{mM}$ & 108 & \\
\hline $1 \mathrm{mM}$ & & \\
\hline & & \\
\hline
\end{tabular}

Polyamine levels change over time in the brains of PS19 mice (33). To further our understanding of how polyamine dysregulation aids in developing tau neuropathology, we tested several mechanisms at the molecular level involving polyamines versus acetylpolyamines, tau aggregation, and tubulin interaction. Our findings identified spermidine and spermine having the greatest effects on tau biology, whereas their acetylated products showed null or opposite tau effects, implicating SSAT as a potential mediator. Spermidine reduced tau fibrillization, oligomerization, and tau seeding/propagation by different methods, whereas acetylspermidine showed reduced or opposite effects. Importantly, spermidine (the median polyamine) showed the greatest abundance of all the polyamines in the brain and possibly offers substrate for both putrescine and spermine when needed. Although spermidine did not affect tubulin polymerization, spermine showed a different profile due to the longer aliphatic chain length and strength of its positive charges. Spermine also reduced tau fibrillization/oligomerization but to a lesser extent affected tau seeding/propagation. However, acetylspermine increased tau fibrilization/oligomerization and promoted tau seeding/propagation.

Spermine also increased tubulin polymerization and bundling but did so at the expense of displacing tau from microtubules. These data are not surprising given that the binding sites of spermine to tubulin (aa residues 430-432 and 444-451) overlap with that of tau and tubulin (aa residues 421-441), providing a possible mechanism by which tau displacement from tubulin could result from increased levels of spermine (34). Additionally, in regard to tubulin binding, spermine and tau binding sites overlap with calcium binding (aa residues 421-443), which is also altered in patients with $\mathrm{AD}$ and mouse models of AD (35). In our studies, spermine sequestered tau in a soluble monomeric state, preventing the accumulation of soluble and insoluble HMW tau species in $\mathrm{C} 3 \mathrm{H} /$ tau cells. Additionally, spermine decreased insoluble monomeric tau in $\mathrm{C} 3 \mathrm{H} / \mathrm{tau}$ cells and prevented tau dimerization/oligomerization in N2a-ssGT cells. Altogether, it remains tempting to speculate that during acute stress, the PSR becomes activated to promote transient polyamine accumulation for neuronal support coupled with end-product removal (acetylpolyamines) after the resolution of stress. However, an alternative outcome suggests that displaced tau is also left vulnerable for fibrillization/oligomerization by the accompanying increase in acetylpolyamines due to SSAT induction. Increased spermidine and spermine were capable of inducing SSAT over time. Ultimately, the effects of acetylpolyamines on tau aggregation within the cell depend on the cell's ability to clear acetylated products. Given that acetylpolyamines affect tau in different capacities (i.e., oligomerization/fibrillization/seeding), efflux of acetylpolyamines (extracellular accumulation) could also render further tau seeding/

A Control
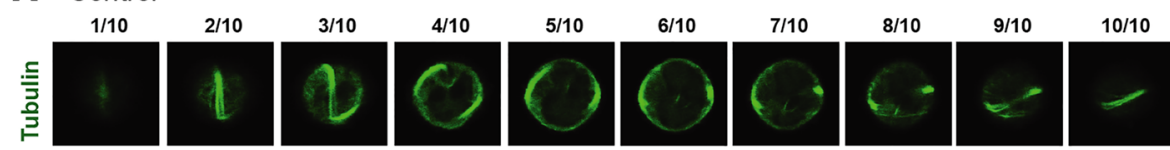

Spermine $0.5 \mathrm{mM}$
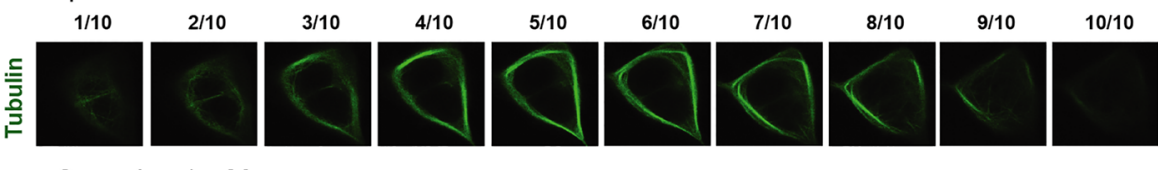

Spermine $1 \mathrm{mM}$
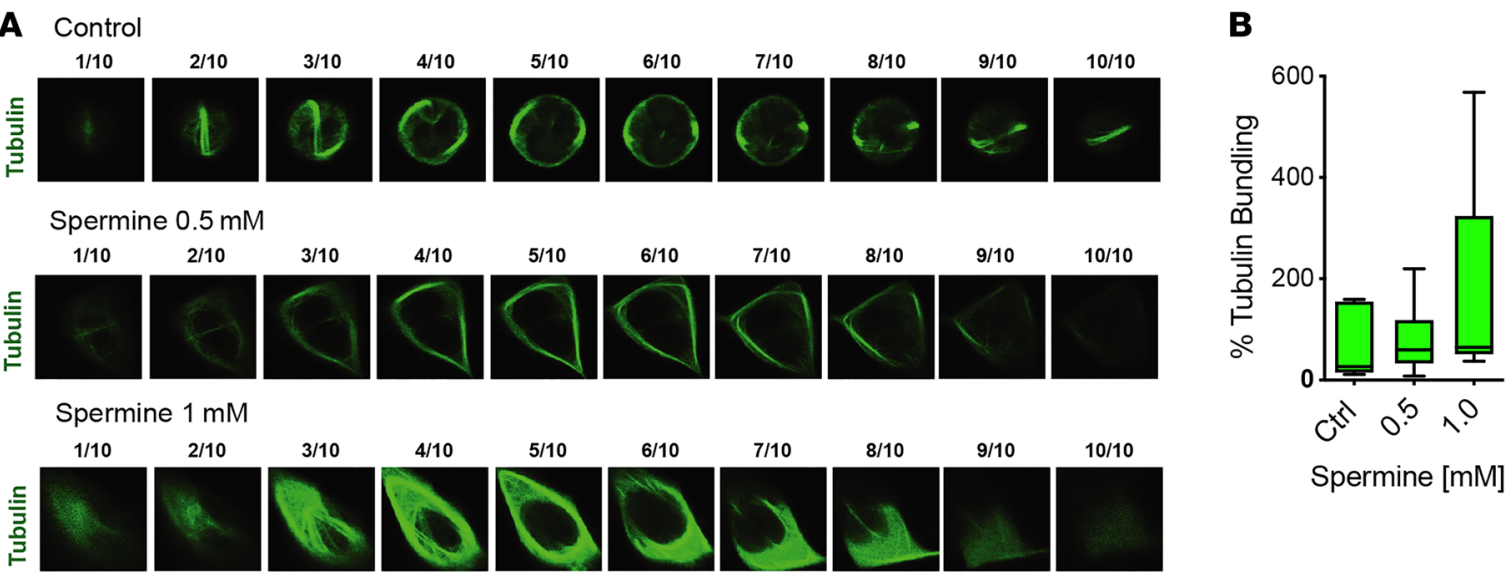

Figure 11. Spermine affects tubulin bundling. (A and B) A 1-way ANOVA indicated no significant effect of treatment on tubulin bundling; however, post hoc analyses showed $1 \mathrm{mM}$ spermine trended toward tubulin bundling $(P=0.087)$ relative to control. One-way ANOVA followed by post hoc analyses using Dunnett's 2-sided correction, $n=7-12$, data were represented by box-and-whisker plot displaying the minimum, first quartile, median, third quartile, and maximum. 
A

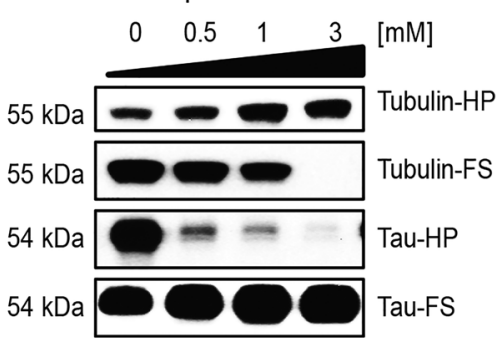

B Tubulin Heavy-Pellet

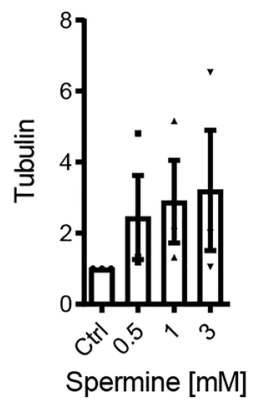

C Tubulin Free-Soluble

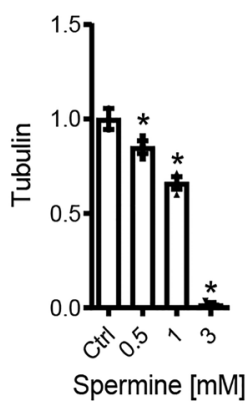

D Tau Heavy-Pellet

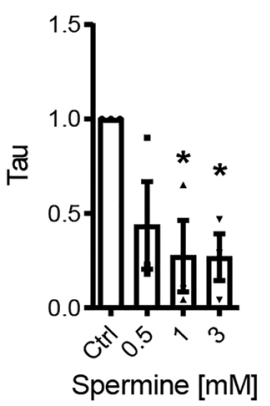

E Tau Free-Soluble

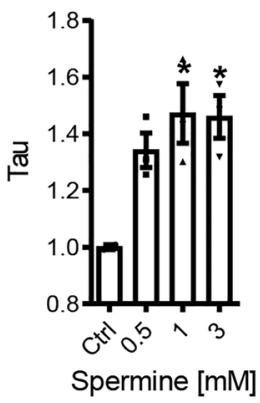

Figure 12. Spermine increases tubulin polymerization and displaces tau. (A) Representative Western blot images of insoluble and soluble fractions of tubulin and tau from a cosedimentation assay. (B-E) A 1-way ANOVA indicated a significant effect of treatment on soluble tubulin, insoluble and soluble tau from spermine treatment $(P=0.000, P=0.039, P=0.022$, respectively). Post hoc analyses indicated that $0.5 \mathrm{mM}, 1 \mathrm{mM}$, and $3 \mathrm{mM}$ decreased soluble tubulin (free soluble) $(P=0.042, P=0.001, P=0.000$, respectively); $1 \mathrm{mM}$ and $3 \mathrm{mM}$ decreased insoluble tau (heavy pellet) $(P=0.033, P=0.032$, respectively); $1 \mathrm{mM}$ and $3 \mathrm{mM}$ increased soluble tau (free soluble) $(P=0.014, P=0.016$, respectively) relative to control. One-way ANOVA followed by post hoc comparisons using Dunnett's 2 -sided correction, $n=3$ ( 2 independent experiments), ${ }^{*} P<0.05$, data represented by mean $\pm \mathrm{SEM}$.

propagation and tau spreading. Although it remains difficult to isolate the exact compartmental concentrations of polyamines versus acetylpolyamines within and outside cells, particularly because of rapid interconversion between various polyamine pools, we demonstrated that targeted disruption of SSAT prevented the accumulation of acetylated byproducts and reduced tau neuropathology (24).

In conclusion, our findings cemented the notion of polyamine dysregulation in $\mathrm{AD}$ brains and demonstrated that altered polyamine dysregulation, through increased AZIN2, precipitated tau neuropathology and induced cognitive and affective impairments. These data also suggest the intriguing notion that altered polyamine status through PSR activation may seed tauopathies and vice versa. We recognized that although AZIN2 was the primary focus in these studies, many of the polyamine transcripts in the $\mathrm{AD}$ brain changed. Importantly, this system is typically self-correcting and tightly regulated. In our animal studies, the presence of tau altered polyamine homeostasis and instead promoted a feed-forward loop by chronically increasing many anabolic enzymes along the entire axis. Further, while polyamines may possess acute stabilizing effects on neuronal function through microtubule homeostasis and prevention of tau fibrillization, the long-term polyamine flux (i.e., recurrence and magnitude) may also contribute to disease progression through
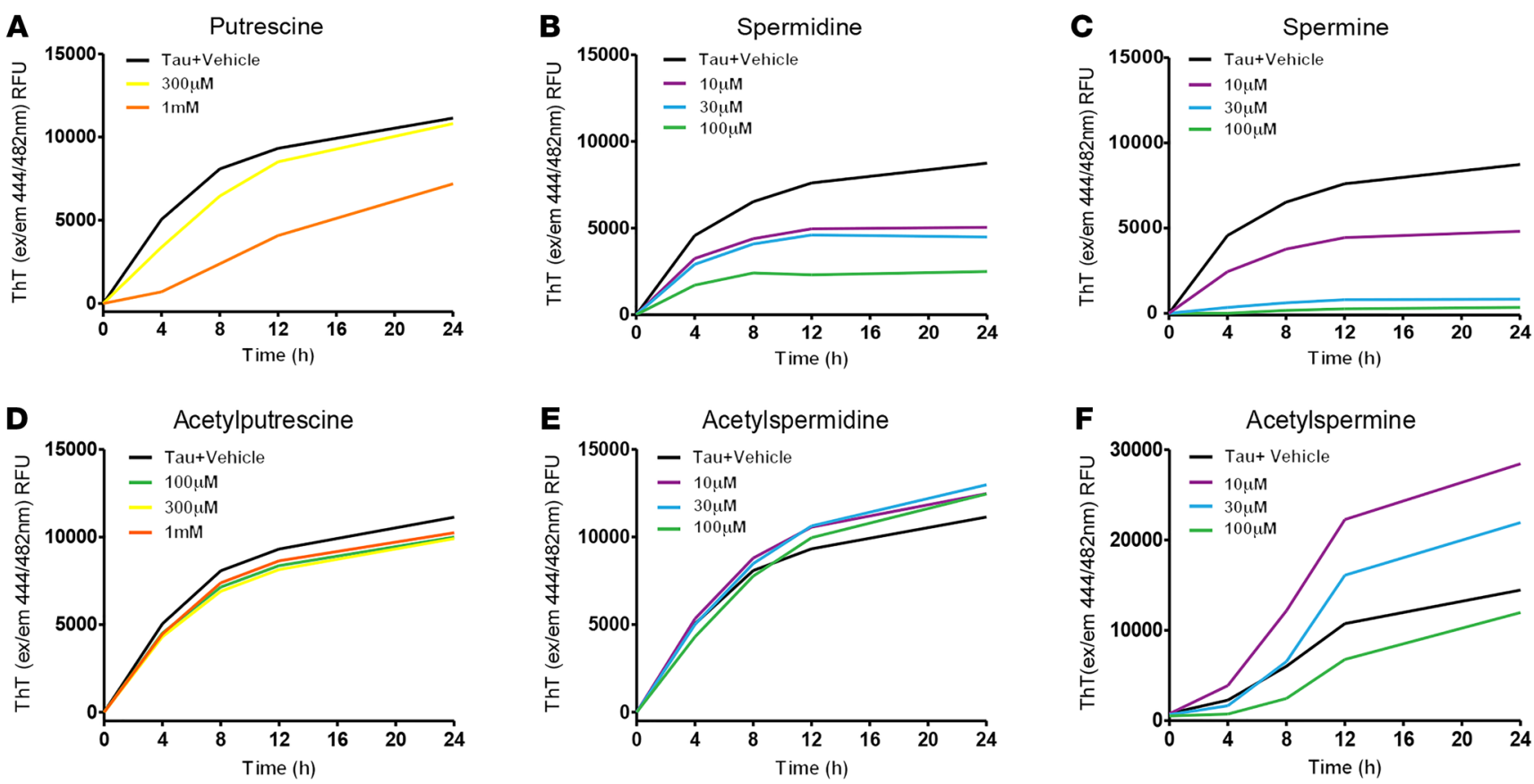

Figure 13. Polyamines and acetylpolyamines differentially affect tau fibrillization. (A-F) Thioflavin T (ThT) assay using recombinant 4RON WT tau and treatment of polyamines (putrescine, A; spermidine, B; spermine, C) or acetylpolyamines [acetylputrescine], D; acetylspermidine, E; acetylspermine, F), followed by quantification of the AUC compared with Tau + Vehicle controls. Data are represented as triplicate averages and Table 3 reflects AUC change from vehicle control (\%Tau + Vehicle, \%Change). 


\section{Table 3. Thioflavin T assay tau aggregation}

\begin{tabular}{|c|c|c|}
\hline Putrescine & \% Tau + Vehicle & $\%$ Change \\
\hline Tau + Vehicle & 100 & \\
\hline $300 \mu \mathrm{M}$ & 89 & -11 \\
\hline $1 \mathrm{mM}$ & 45 & -55 \\
\hline Spermidine & \% Tau + Vehicle & $\%$ Change \\
\hline Tau + Vehicle & 100 & \\
\hline $10 \mu \mathrm{M}$ & 64 & -36 \\
\hline $30 \mu \mathrm{M}$ & 58 & -42 \\
\hline $100 \mu \mathrm{M}$ & 32 & -68 \\
\hline Acetylputrescine & \% Tau + Vehicle & $\%$ Change \\
\hline Tau + Vehicle & 100 & \\
\hline $100 \mu \mathrm{M}$ & 90 & -10 \\
\hline $300 \mu \mathrm{M}$ & 87 & -13 \\
\hline $1 \mathrm{mM}$ & 92 & -8 \\
\hline Acetylspermidine & \% Tau + Vehicle & $\%$ Change \\
\hline Tau + Vehicle & 100 & \\
\hline $10 \mu \mathrm{M}$ & 111 & 11 \\
\hline $30 \mu \mathrm{M}$ & 112 & 12 \\
\hline $100 \mu \mathrm{M}$ & 105 & 5 \\
\hline Spermine & \% Tau + Vehicle & $\%$ Change \\
\hline Tau + Vehicle & 100 & \\
\hline $10 \mu \mathrm{M}$ & 57 & -43 \\
\hline $30 \mu \mathrm{M}$ & 10 & -90 \\
\hline $100 \mu \mathrm{M}$ & 3 & -97 \\
\hline Acetylspermine & \% Tau + Vehicle & $\%$ Change \\
\hline Tau + Vehicle & 100 & \\
\hline $10 \mu \mathrm{M}$ & 200 & 100 \\
\hline $30 \mu \mathrm{M}$ & 142 & 42 \\
\hline $100 \mu \mathrm{M}$ & 67 & -33 \\
\hline
\end{tabular}

indirect mechanisms, such as tau displacement and accumulation of acetylated byproducts to facilitate disease progression. These data highlight a potentially novel interaction between tau neuropathology, polyamine regulation, and behavioral impairments and suggests potentially new therapeutic strategies along the polyamine axis to treat tauopathies, including AD.

\section{Methods}

Human hippocampal brain tissue. For qRT-PCR analyses, the Institute for Brain Aging and Dementia Tissue Repository at the University of California (Irvine) provided human postmortem hippocampal tissue from AD and control patients. Pathological and clinical criteria confirmed the diagnosis of $\mathrm{AD}$. Tissue was frozen at autopsy and stored at $-80^{\circ} \mathrm{C}$ until use. Control and $\mathrm{AD}$ samples were matched for age and gender. RNA was extracted as previously described (36) and was analyzed by qRT-PCR. Eight total control samples ( 2 male, 6 female) and 10 AD samples (4 male, 6 female) were analyzed for gene transcripts. To accurately match for age/gender across PCR-array plates (which included 1 control and $1 \mathrm{AD}$ sample), 2 male control samples were used twice; however, their re-run data was not included in analyses. A table depicting the distribution of age, gender, and postmortem interval (PMI) of qRT-PCR samples can be found in Supplemental Tables 1-3.

For Western blot analyses, samples were deidentified and obtained from the NIH NeuroBioBank following the institutional bioethics guide- lines. Brain (cortex) from cases of neuropathologically confirmed $\mathrm{AD}(n$ $=15$; age range 67-100; $[81.8 \pm 1.8$, mean \pm SEM] $)$ and normal cases $(n=$ 10 ; age range 69-89; $[78.5 \pm 2.4$, mean \pm SEM] $)$ were obtained. The average PMI was $(\mathrm{AD}=14.9 \pm 2.1$, mean $\pm \mathrm{SEM} ; \mathrm{C}=16.1 \pm 2.2$, mean $\pm \mathrm{SEM})$. Samples were categorized for sex, Braak Staging, and phospho-tau positivity (tau AT8 positive or negative, Supplemental Tables 4-6).

qRT-PCR. The cDNA from human samples, used with $\mathrm{RT}^{2} \mathrm{SYBR}$ Green qPCR Mastermix (Qiagen, 330529), was performed using a custom RT ${ }^{2}$ Profiler PCR array (Qiagen, 330171), according to the manufacturer's protocol. CT values exported to an Excel file were uploaded to the analysis web portal at http://www.qiagen.com/geneglobe. Samples were assigned to controls or test groups. CT values were normalized based on a manual selection of reference genes. The data analysis web portal calculates the fold change/regulation using the $\Delta \Delta \mathrm{CT}$ method, in which $\Delta \mathrm{CT}$ is calculated between the gene of interest and an average of housekeeping genes, followed by $\Delta \Delta \mathrm{CT}$ calculations ( $\Delta \mathrm{CT}$ [experiment] - $\Delta$ CT [control]). Fold-change was calculated using the $2^{-\Delta \Delta C T}$ formula. The data analysis web portal generated a scatter plot, heatmap, hierarchy clustergram analysis, and multigroup plot data analysis. Each 96-well plate contained 43 disease-focused transcripts and 2 housekeeping genes per sample (1 control and $1 \mathrm{AD}$ sample per plate). In addition, 1 well contained a genomic DNA control, 1 well contained reverse-transcription controls, and 1 well contained positive PCR controls per sample, all of which passed quality control metrics. In this study, we report a total of 47 genes profiled on 18 samples.

Mice. All animal procedures were performed following the IACUC at the University of South Florida Health Byrd Alzheimer's Institute. Male and female nTg (C57BL/6J, 000664) and tau PS19 transgenic mice [Tg(Prnp-MAPT ${ }^{*}$ P301S)PS19Vle, The Jackson Laboratory, 008169] were used, and experimental group assignments were balanced for genotype, gender, and litter (37).

Viral production. AZIN2 was cloned into the rAAV vector pTR2MCS. This vector expressed the AZIN2 with the chicken $\beta$-actin CMV hybrid promoter and contained the AAV2 terminal repeats. The AZIN2 was also appended on the $\mathrm{N}$-terminus with an HA tag to detect the expressed protein. $\mathrm{rAAV}$ serotype 9 viral particles were generated from a triple transfection into HEK293 cells (ATCC, CRL-1573), followed by purification as described previously (38).

Surgical procedures. At 4 months of age, nTg and tau PS19 mice were anesthetized with isoflurane, and a volume of $2 \mu \mathrm{L}$ of AAV particles containing AVV9-empty capsid (EC) or AZIN2 $\left(3 \times 10^{12}\right.$ $\mathrm{vg} / \mathrm{mL}$ ) were stereotaxically injected bilaterally into the CA3 of the hippocampus and the anterior cortex. Stereotaxic coordinates from bregma were $\mathrm{AP}=-2.5 \mathrm{~mm}, \mathrm{ML}= \pm 2.9 \mathrm{~mm}$, and $\mathrm{DV}=-3.0 \mathrm{~mm}$ for the hippocampus, and $\mathrm{AP}=2.2 \mathrm{~mm}, \mathrm{ML}= \pm 1.7 \mathrm{~mm}$, and $\mathrm{DV}=$ $-3.0 \mathrm{~mm}$ for the cortex. The virus was administered using convection-enhanced delivery at a constant rate of $1.5 \mu \mathrm{L} / \mathrm{min}$ (39). Viral incubation was 4 months at the start of behavioral testing and was 5 months at the time of euthanasia and tissue collection.

Behavioral testing. Four months after viral incubation, mice began behavioral tasks to assess alterations in affective processing, cognitive performance, and motor performance. Behavioral tasks were run in sequence to minimize stress effects on behavioral performance and were adequately spaced out over a month to prevent carryover effects on performance. White noise $(55 \mathrm{~dB})$ was present during all testing. The experimenter was blinded to the sample group allocation during data collection. 
A Putrescine [mM]

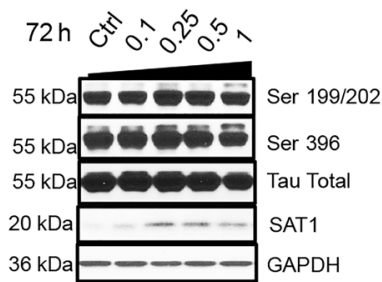

B Spermidine $[\mathrm{mM}]$

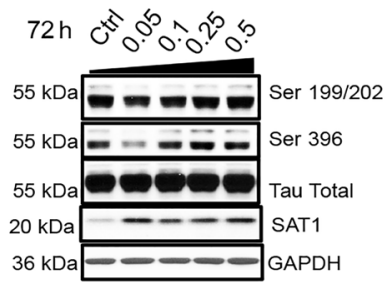

C Spermine $[\mathrm{mM}]$

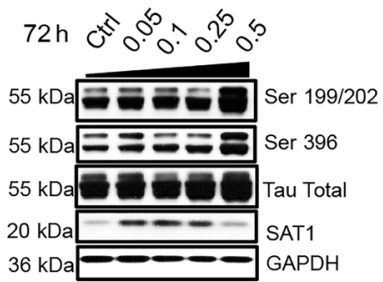

D

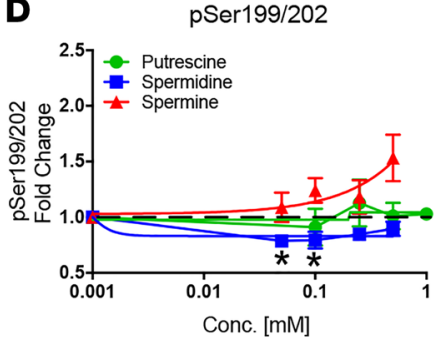

H

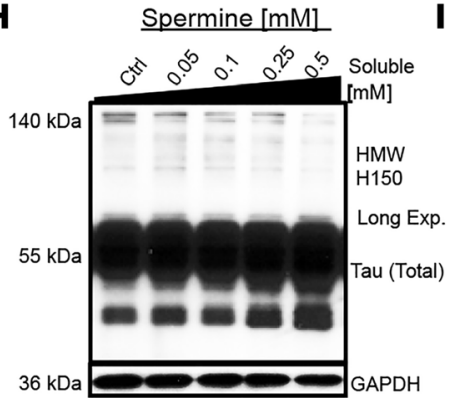

E

I
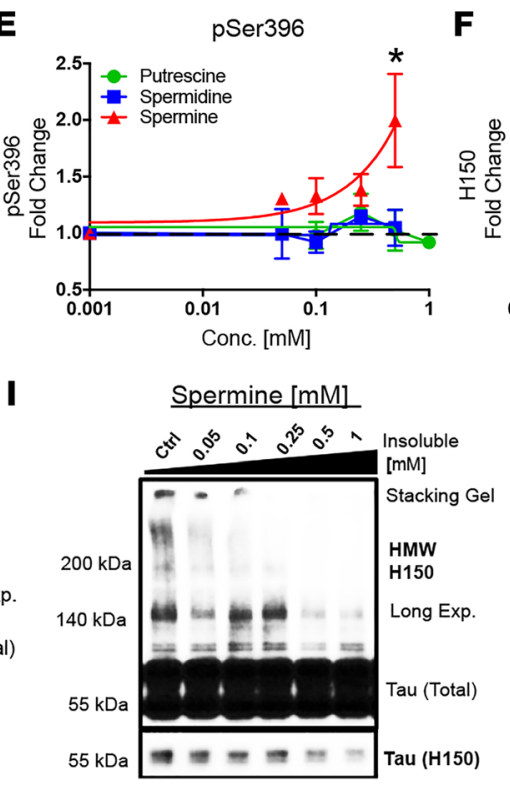

F

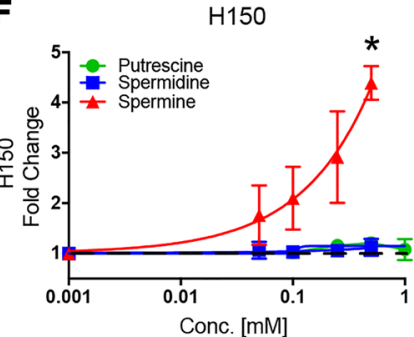

G

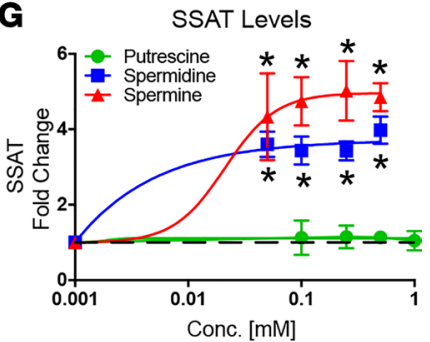

K

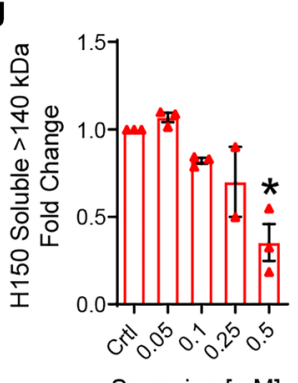

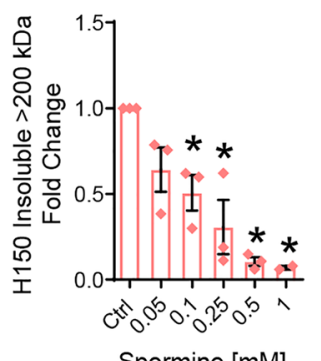
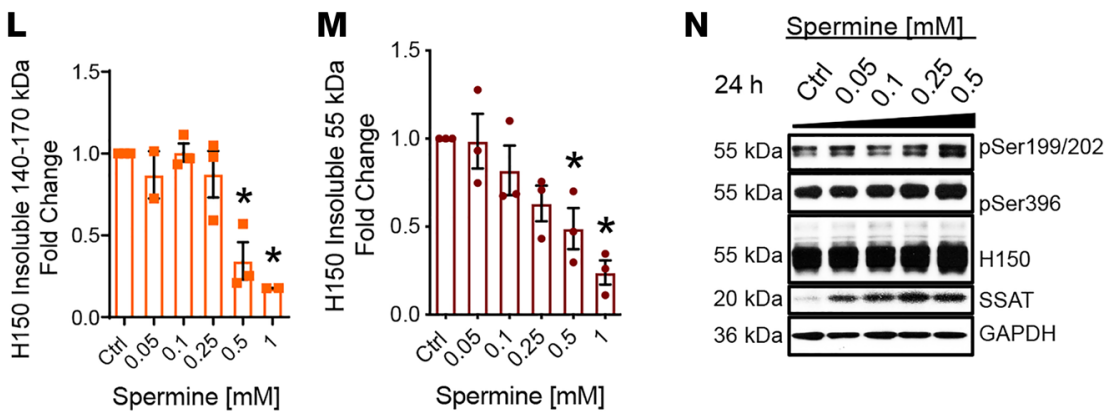

- Protein Expression (24h)

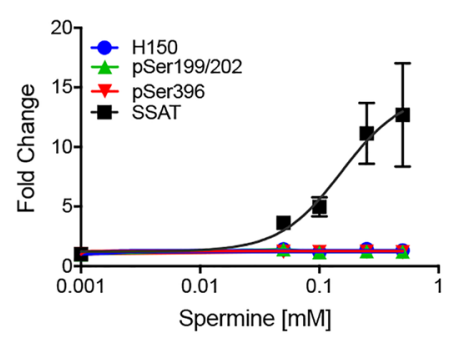

Figure 14. Spermine sequesters tau in monomeric state, preventing higher-order aggregation, in tau-overexpressing cells. (A-C) Representative Western blot images of $\mathrm{C} 3 \mathrm{H} /$ tau cells; 72 hours. (D-G) Spermidine $(0.05-0.1 \mathrm{mM})$ decreased $\mathrm{pS199} / 202(P=0.036, P=0.043$, respectively), and (0.05-0.5 mM) increased SSAT $(P=0.000, P=0.001, P=.001, P=0.000$, respectively). Spermine $(0.5 \mathrm{mM})$ increased tau pS396 $(P=0.023)$, tau H150 $(P=0.019)$, and $(0.05,0.1,0.25$, and $0.5 \mathrm{mM})$ SSAT $(P=0.033, P=0.018, P=0.012$, and $P=0.028$, respectively). (H and $\mathrm{I})$ Representative Western blot images of $\mathrm{C} 3 \mathrm{H} /$ tau cells. (J) Spermine $(0.5 \mathrm{mM})$ reduced soluble total tau $(\mathrm{H} 150 ; P=0.001)$. (K) Spermine $(0.1,0.25,0.5,1.0 \mathrm{mM})$ reduced insoluble HMW ( $>200 \mathrm{kDa}$ ) total tau (H150; $P=0.0186, P=0.002, P=0.000, P=0.000$, respectively). (L) Spermine (0.5-1.0 mM) reduced insoluble oligomeric (140-170 kDa) total tau (H150; $P=0.002, P=0.001$, respectively). (M) Spermine (0.5-1.0 mM) reduced insoluble tau (55 kDa) (H150; $P=$ $0.025, P=0.002$, respectively). (N) Representative Western blot images of C3H/tau cells. ( 0 ) Spermine (0.5 mM) trended toward increased SSAT expression $\left(P=0.087\right.$ ). One-way ANOVA followed by Dunnett's 2 -sided correction, $n=3$ triplicates ( 3 independent experiments), ${ }^{*} P<0.05$, data represented by mean \pm SEM.

Open field. The open-field test was used to assess general locomotor and anxiety-like behavior. Animals were allowed to freely explore the maze for 15 minutes in a $44 \mathrm{~cm}^{2}$ open field while being monitored by video tracking software (AnyMaze software; Stoelting Company). Activity levels were evaluated by total distance traveled. Anxiety was assessed by the number of entries to the center zone and time spent in the apparatus's center zone. Animals that show a decreased tendency to explore in the center of the field can be indicative of a higher anxiety level.
Y maze. The Y maze was used to assess working memory. Animals were allowed to freely explore the maze for 5 minutes while being monitored by video tracking software. Maze measurements were as follows: $35 \mathrm{~cm} \times 5 \mathrm{~cm} \times 10 \mathrm{~cm}(\mathrm{~L} \times \mathrm{W} \times \mathrm{H})$. Alternation was calculated as the percentage of successful alternations (entering each arm at least once in 3 attempts) of total possible alternations (number of arm entries minus 2). Animals performing a decreased number of alternations is indicative of impaired working memory. 

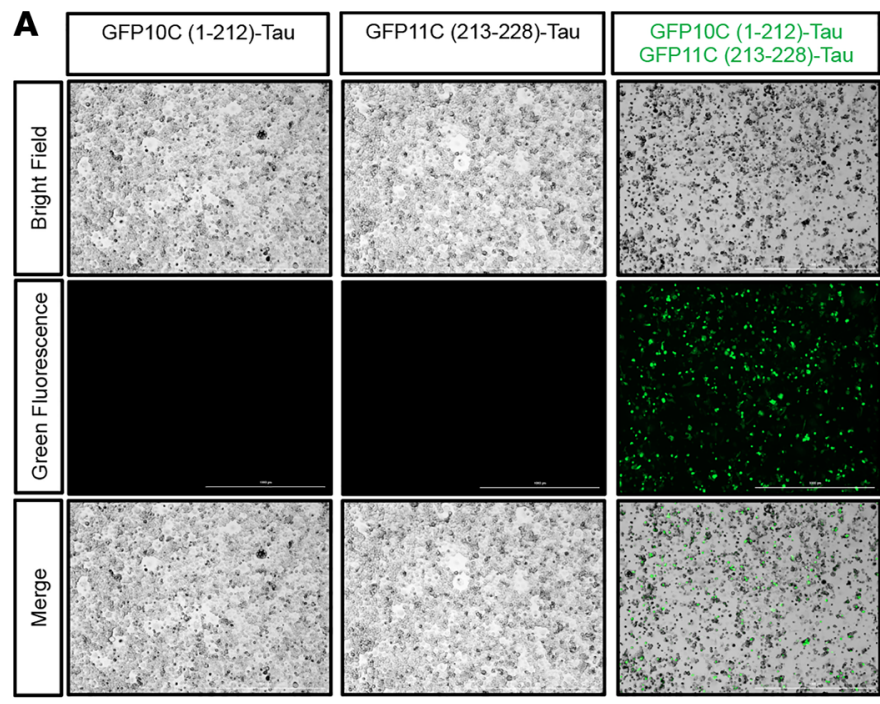

Scale bar: $1000 \mu \mathrm{m}$
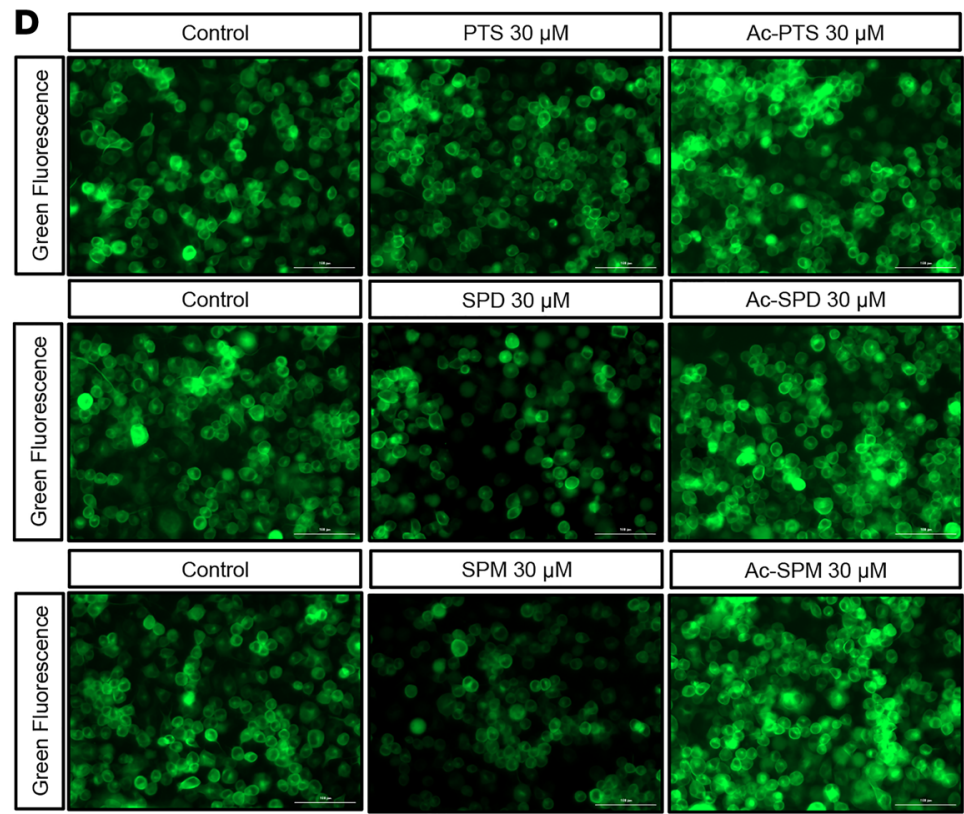

Scale bar: $100 \mu \mathrm{m}$
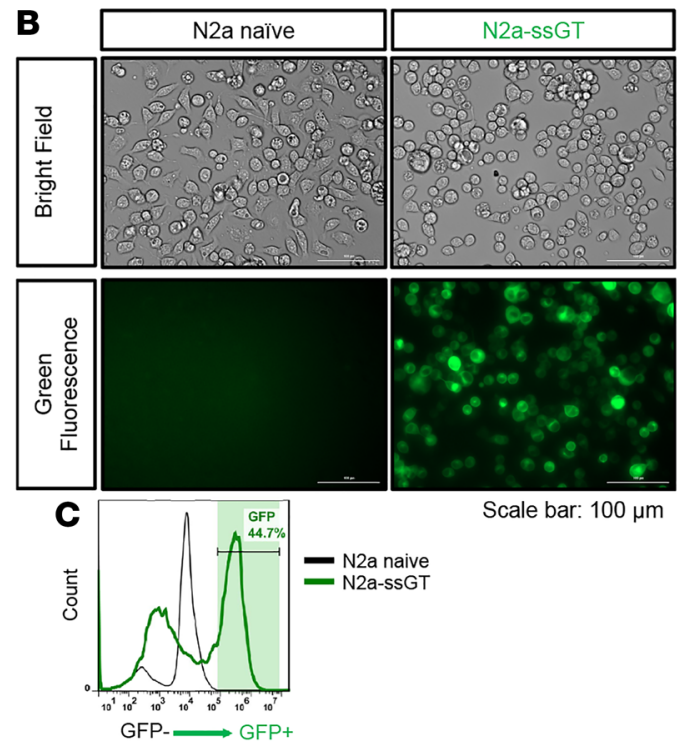

Scale bar: $100 \mu \mathrm{m}$
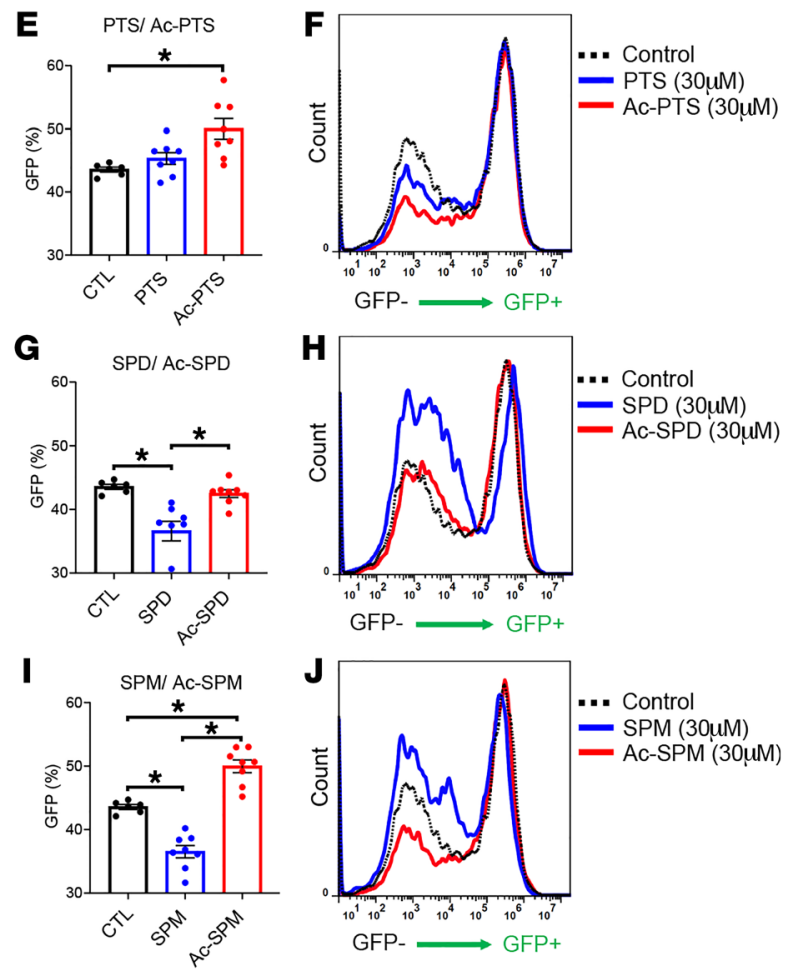

Figure 15. Polyamines decrease tau oligomerization while acetylpolyamines increase tau oligomerization. (A-C) Validation of split GFP-Tau plasmids (pmGFP10C-Tau, pmGFP11C-Tau) and a monoclonal cell line (N2a-SSGT). (D) Representative images of tau oligomerization after treatment with polyamines/ acetylpolyamines for 72 hours. Original magnification, $\times 10$. (E-J) Graphs and histograms show the effect of treatment on tau oligomerization, as measured by percentage GFP $(P=0.000)$. Spermidine and spermine $(30 \mu \mathrm{M})$ decreased tau oligomerization compared with controls $(P=0.003, P=0.002$, respectiveIy), while acetylputrescine and acetylspermine increased tau oligomerization $(P=0.007, P=0.007$, respectively) compared with controls. PTS: putrescine, Ac-PTS: acetylputrescine, SPD: spermidine, Ac-SPD: acetylspermidine, SPM: spermine, Ac-SPM: acetylspermine. Acetylputrescine (30 $\mu M)$ trended toward increased tau oligomerization $(P=0.062)$ compared with $30 \mu \mathrm{M}$ putrescine. Acetylspermidine $(30 \mu \mathrm{M})$ increased tau oligomerization $(P=0.008)$ compared with $30 \mu \mathrm{M}$ spermidine. Acetylspermine $(30 \mu \mathrm{M})$ increased tau oligomerization $(P=0.000)$ compared with $30 \mu \mathrm{M}$ spermine. One-way ANOVA followed by post hoc comparisons using Tukey's HSD, $n=6-8,{ }^{*} P<0.05$; data represented by mean \pm SEM.

Inhibitory avoidance. The inhibitory avoidance test was used to assess inhibition and fear-associated recall based on the association between a specific environmental context, which the animal learns to avoid, and an aversive stimulus represented by a mild foot shock. The testing apparatus was divided into 2 compartments by a partition with a sliding door. On day 1 , the animal was placed into the brightly lit side of the chamber; after 30 seconds the door opened to the dark chamber and upon entering the dark chamber, the mice received a mild foot shock ( $0.5 \mathrm{~mA}, 1$ second). On day 2, the animals were placed in the brightly lit chamber and after 30 
A
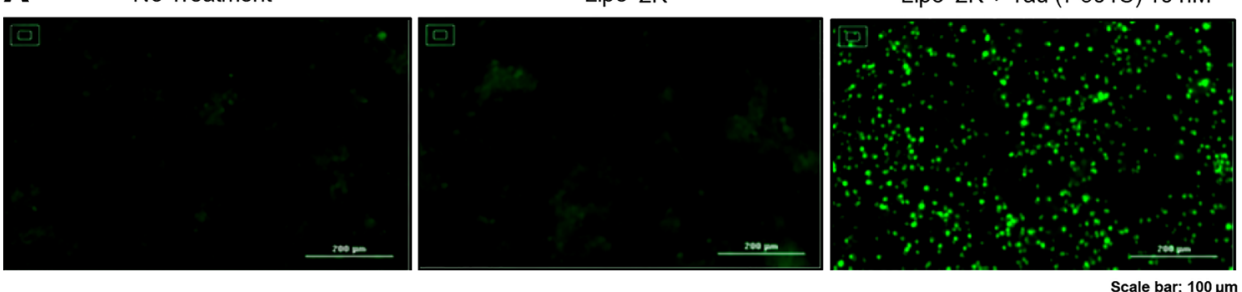

Scale bar: $100 \mu \mathrm{m}$

C

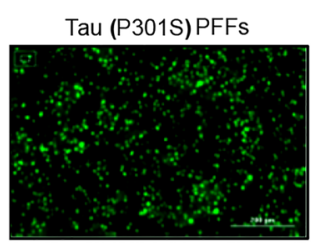

E

Tau (P301S) PFFs

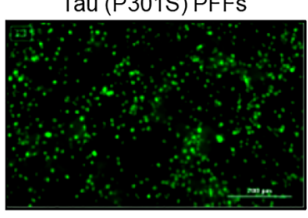

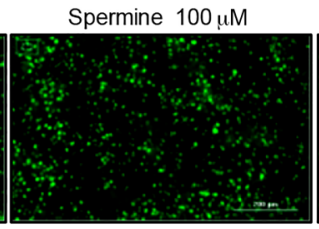

Acetylspermine $30 \mu \mathrm{M}$

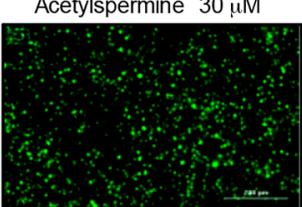

Spermine $30 \mu \mathrm{M}$

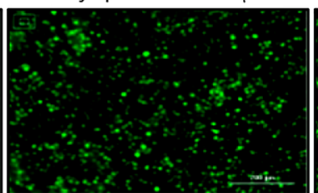

Spermidine $30 u \mathrm{M}$

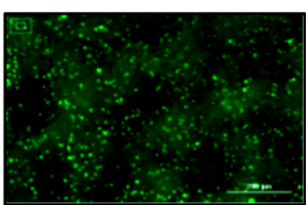

Acetylspermidine $30 \mu \mathrm{M}$
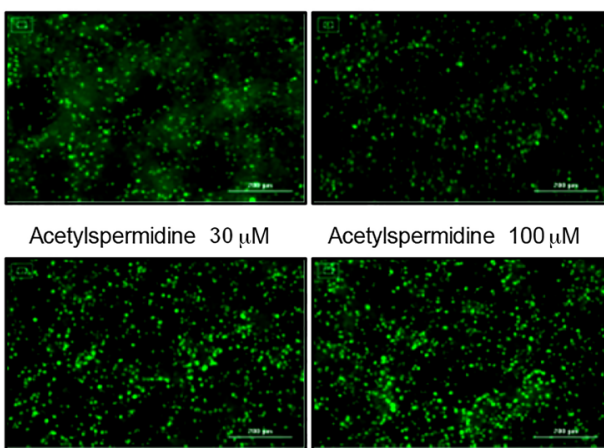

Acetylspermidine $100 \mu \mathrm{M}$

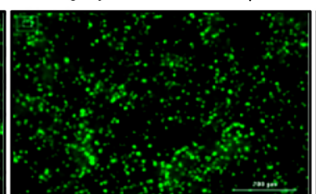

Spermidine $100 \mu \mathrm{M}$

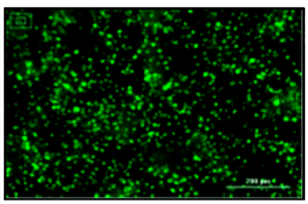

Scale bar: $100 \mu \mathrm{m}$

Spermine $300 \mu \mathrm{M}$

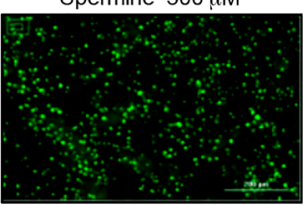

Acetylspermine $300 \mu \mathrm{M}$

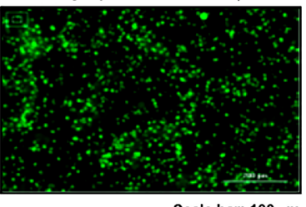

B

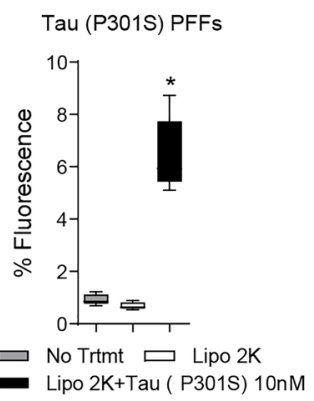

Scale bar: $100 \mu \mathrm{m}$

Tau (P301S) PFFs (10nM) + + + + + +

Spermidine $(\mu \mathrm{M})$ - $30100300-\quad-$ Acetylspermidine $(\mu \mathrm{M}) \quad$ - - - $\quad 30100300$

F Spermine/Acetylspermine

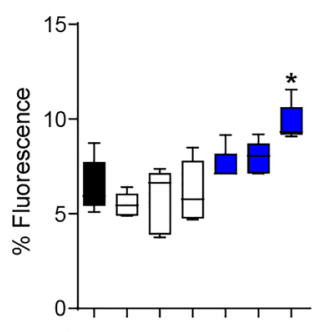

Tau (P301S) PFFs (10nM) + + + + + +

Spermine $(\mu \mathrm{M})$ - 30100300 - - -

Acetylspermine $(\mu \mathrm{M})$ - - - 30100300

Figure 16. Polyamines decrease tau seeding while acetylpolyamines increase tau seeding. (A and B) Validation of tau seeding aggregation assay using TauRD P301S FRET biosensor cells by $10 \mathrm{nM}$ active human recombinant tau441 (2N4R), P301S mutant tau preformed fibrils (Tau [P301S] PFFs), $P=0.000$. Lipofectamine $2000+10 \mathrm{nM}$ Tau PFFs increased seeding compared with no treatment and Lipofectamine 2000 alone $(P=0.000, P=0.000$, respectively) (C and D) There was a significant effect of treatment on tau aggregation as measured by percentage fluorescence $(P=0.000)$. Spermidine $(300 \mu \mathrm{M})+10$ nM Tau (P3015) PFFs decreased tau aggregation $(P=0.021)$, while acetylspermidine $(300 \mu \mathrm{M})+10 \mathrm{nM}$ Tau (P301S) PFFs increased tau aggregation $(P=$ 0.008) compared with $10 \mathrm{nM}$ Tau (P3015) PFFs alone. (E and F) There was a significant effect of treatment on tau aggregation as measured by percentage fluorescence $(P=0.000)$. Acetylspermine $(300 \mu \mathrm{M})$ increased tau aggregation $(P=0.003)$. One-way ANOVA followed by post-hoc comparisons using Tukey's HSD, $n=5$, data represented by box-and-whisker plot displaying the minimum, first quartile, median, third quartile, and maximum; ${ }^{*} P<0.05$.

seconds the door opened to the dark chamber. Latency to cross into the dark chamber was characterized as basal inhibition (day 1) and fear-associated recall (day 2). On day 1 , increased latency indicated increased inhibition. On day 2, decreased latency indicated impaired fear-associated recall. On both days, the maximum latency to cross was 300 seconds or 5 minutes.

Immunohistochemistry and staining. After completing the behavioral testing battery, mice were euthanized with SomnaSol and transcardially perfused with $0.9 \%$ saline at 9 months of age. One hemisphere of the brain was dissected, frozen, and stored at $-80^{\circ} \mathrm{C}$ for biochemical analysis; the opposite hemisphere was fixed in $4 \%$ paraformaldehyde in $100 \mathrm{mM}$ phosphate buffer (pH 7.4) for 24 hours. Tissue was cryopro- tected by sequential immersion in 10\%, $20 \%$, and $30 \%$ sucrose solutions for 24 hours each. Brains were sectioned at $25 \mu \mathrm{m}$ using a sliding microtome and stored at $4^{\circ} \mathrm{C}$ in Dulbecco's PBS containing $100 \mathrm{mM}$ sodium azide until staining. Immunohistochemistry was performed on free-floating sections (6-8 per mouse) as previously described (40) and stained for the AZIN2 viral HA tag (Roche, 12158167001), total human tau (HT7; Thermo Fisher Scientific, MN1000B), PHF (AT8, Thermo Fisher Scientific, MN1020B), and microglial activation (IBA1; Wako Chemicals USA, Inc., 016-26461).

Polyamine quantification. Polyamine quantification in brain homogenates was performed in collaboration with Sanford Burnham Prebys. Total brain homogenates were subjected to liquid chro- 
A

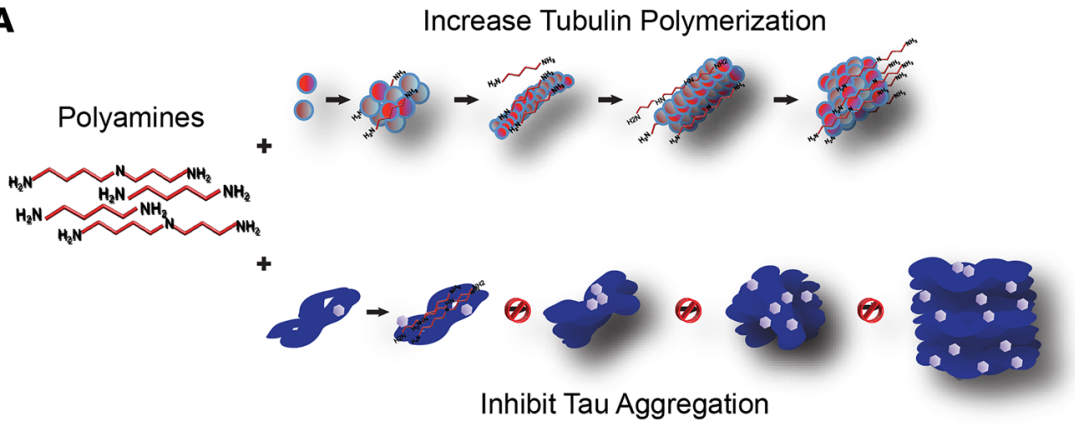

B

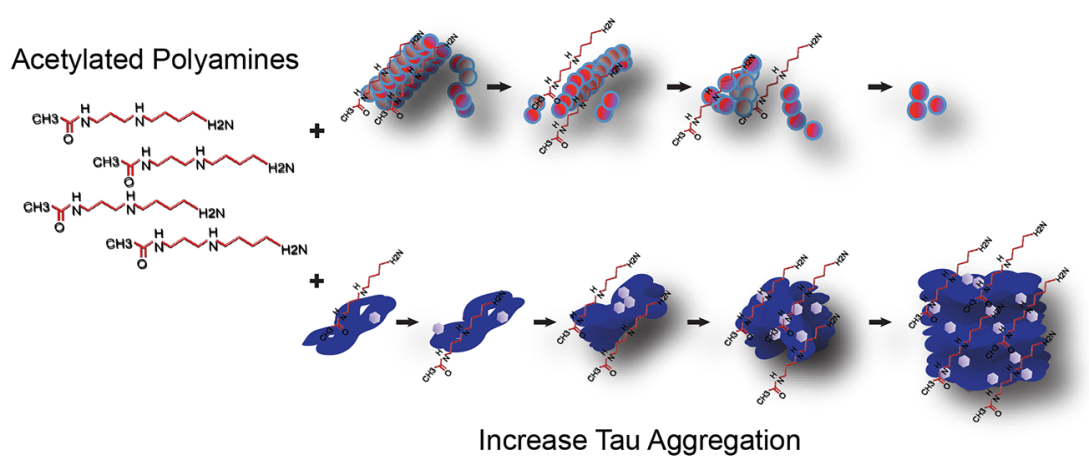

C

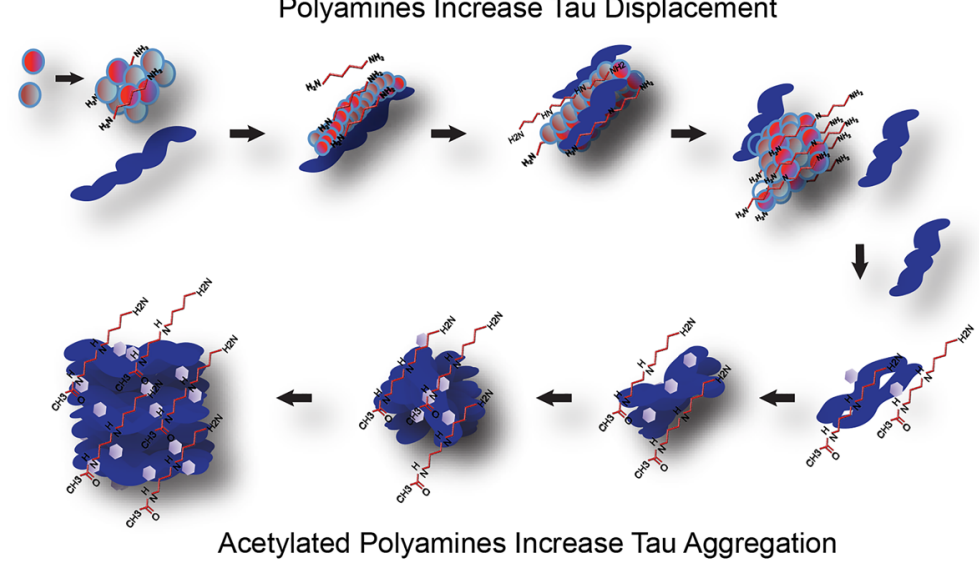

Figure 17. Hypothesized interactions of polyamines, tubulin, and tau. (A) Polyamines increase tubulin polymerization while also inhibiting tau aggregation. (B) Acetylpolyamines fail to increase and even reduce tubulin polymerization and promote tau fibrillization and aggregation. (C) In tauopathies, increased levels of polyamines facilitate tubulin polymerization but displace tau, leaving it vulnerable for aggregation by increased levels of acetylpolyamines.

93482) on ice. Tissues were homogenized in $20 \%(\mathrm{w} / \mathrm{v})$ consisting of the buffer mentioned above, and an aliquot was centrifuged at $14,000 \mathrm{~g}$ for 10 minutes at $4^{\circ} \mathrm{C}$. The supernatant (S1) was transferred to another tube and subjected to BCA assay protein quantification. Approximately $20 \mu \mathrm{g}$ of protein was resolved using $4 \%-20 \%$ gradient gel, transferred to PVDF, and probed for Tau5 (MilliporeSigma, MAB361), PHF Tau (AT8; Thermo Fisher Scientific, MN1020), AZIN2/ADC (Abcam, ab157214), and GAPDH (Meridian, H86504 male). After 3 TBS washes in $0.05 \%$ Tween 20 , appropriate secondary antibodies (Southern Biotechnologies, goat anti-rabbit [4010-05], goat anti-mouse [1070-05]) were used and detected with chemiluminescence (Thermo Fisher Scientific, P32106). Images were taken using the Azure Series C600 digital imager. All samples were normalized to their respective actin or GAPDH, and then calculated for fold change.

For mouse brain samples, Western blot analysis was performed as previously described (40). Briefly, dissected hippocampal tissue was weighed and resuspended at $10 \%(\mathrm{w} / \mathrm{v})$ in modified RIPA buffer (50 mM Tris pH 7.6, $140 \mathrm{mM}$ $\mathrm{NaCl}, 1 \% \mathrm{NP}-40,0.5 \% \mathrm{Na}$ deoxycholate, $0.1 \%$ SDS) with a $1 \%(\mathrm{v} / \mathrm{v})$ protease inhibitor cocktail (Sigma-Aldrich, P8340) and phosphatase inhibitor cocktails 2 and 3 (Sigma-Aldrich, P5726 and P0044). Tissue was homogenized with a tissue homogenizer and centrifuged at 40,000 $g$ for 30 minutes at $4^{\circ} \mathrm{C}$. The supernatant containing the detergent-soluble fraction (S1) was analyzed by Western blot. The pellet (P1) containing the insoluble material was resuspended in $70 \%$ formic acid (20\% volume of RIPA buffer used for homogenization) and incubated for 1 hour at room temperature. Formic acid was buffered with $1 \mathrm{M}$ Tris, $\mathrm{pH}$ 7.5 (3 times the volume of $70 \%$ formic acid used for pellet resuspension), and $\mathrm{pH}$ was adjusted with $\mathrm{NaOH}$ solution $(50 \% \mathrm{w} / \mathrm{w})$ added to a final $\mathrm{pH}$ of 7.5. Protein concentration was determined with the Pierce BCA protein assay (Thermo Fisher Scientific, P123225). Five micrograms of total protein (for S1 and P1) were loaded to measure

matography with tandem mass spectrometry (LC-MS/MS) using a standard curve of polyamine analytes. Metabolites not quantified, such as acetylputrescine and acetylspermine, fell below the quantitation limit and are therefore not reported.

Biochemical analysis, Western blotting, and dot blot. For human brain samples, frozen tissue (10-20 mg) was weighed in $1.5 \mathrm{~mL}$ tubes and submerged in RIPA buffer (50 mM Tris- $\mathrm{HCl}, 150 \mathrm{mM} \mathrm{NaCl}, 1 \%$ NP-40, 0.1\% SDS, $0.5 \%$ sodium deoxycholate, $\mathrm{pH}$ 7.4) with protease inhibitor cocktail (Sigma-Aldrich, P8340-5ML), phosphatase inhibitor cocktail 2 (Sigma-Aldrich, P5726-5ML), phosphatase inhibitor cocktail 3 (Sigma-Aldrich, P0044-5ML), and PMSF (Sigma-Aldrich, tau epitopes, including tau total (H150; Santa Cruz Biotechnology, sc-5587), tau AT8 (Thermo Fisher Scientific, MN1020), tau pSer396 (AnaSpec, 54977-025), and tau pSer199/202 (AnaSpec, 54963-025). Five to 10 micrograms of total protein were loaded to measure polyamine enzymes: AZIN2 (Abcam, ab157214), ODC (Epitomics/Abcam, S2610), SRM (Proteintech Group, 19858-1-AP), SMS (Epitomics/ Abcam, ab156879), SMOX (Proteintech Group, 15052-1-AP), SSAT (Abcam, ab105220), and PAOX (Proteintech Group, 189721-1-AP). The primary antibodies used for the cosedimentation assay Western blot analyses were $\beta$-tubulin 9F3 (Cell Signaling Technology, 2128) and total tau (H150; Santa Cruz Biotechnology, sc-5587). 


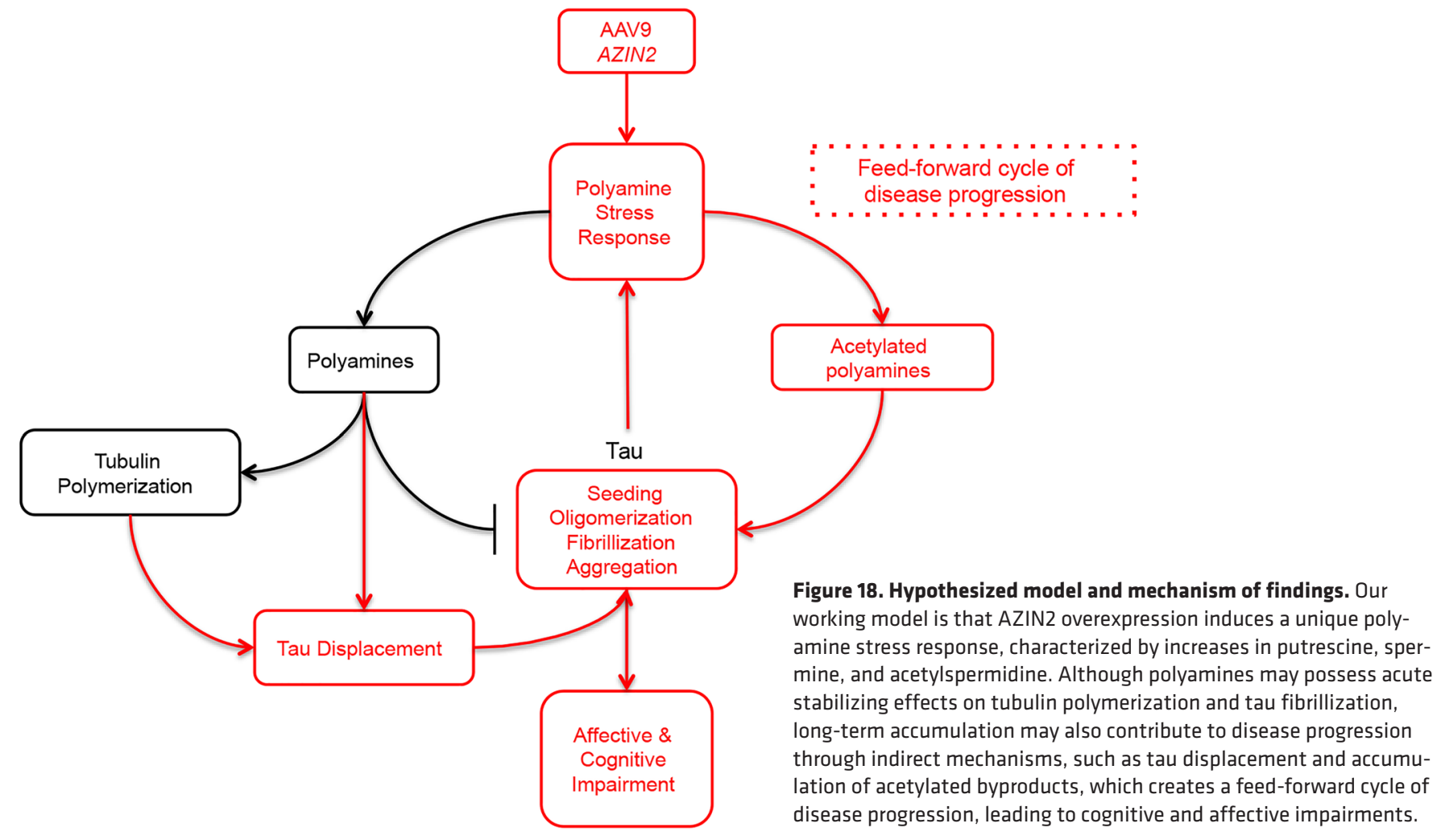

For dot blot analysis, $30 \mu \mathrm{g}$ of total protein from whole-cell homogenates was spotted onto wet nitrocellulose membrane and allowed to air dry. Membranes were washed with TBST (20 mM Tris, $0.8 \% \mathrm{NaCl}, \mathrm{pH} 7.4$, with $0.07 \%$ Tween 20 ), blocked with Blotto in TBS (Thermo Fisher Scientific, 137530) for 30 minutes, washed 3 times for 10 minutes in TBST, and allowed to incubate with the primary antitau oligomeric antibody (T22; MilliporeSigma, ABN454-1) overnight at $4^{\circ} \mathrm{C}$, followed by three 10-minute washes. An HRP-conjugated secondary antibody (Southern Biotechnologies goat anti-rabbit, 401005) was allowed to incubate for 2 hours. Then the membranes were washed 3 times for 10 minutes in TBST and developed using Pierce ECL Western blotting substrate (P32106) and LabScientific Autorad blue film to detect the chemiluminescence signal.

Treatment of C3tau HeLa cells with polyamines. C3tau HeLa $(\mathrm{C} 3 \mathrm{H} / \mathrm{tau})$ cells stably overexpressing human WT 4RON tau were cultured in 100-mm dishes using OPTIMEM media containing $10 \%$ FBS, 100 units $/ \mathrm{mL}$ penicillin, $100 \mu \mathrm{g} / \mathrm{mL}$ streptomycin, amphotericin B, and $0.3 \mu \mathrm{g} / \mathrm{mL} \mathrm{G}-418$. Cells were grown to $90 \%$ confluence and treated with putrescine, spermidine, and spermine (0-1 mM) (Sigma-Aldrich, P5780, S4139, and S4264) for 72 hours in the presence of $1 \mathrm{mM}$ aminoguanidine (Sigma-Aldrich, 396494). For specific experiments, cells were treated for 24 hours under the same conditions. Cells were washed in PBS and harvested for Western blot analysis.

Cells were washed in PBS, collected, and lysed with modified RIPA buffer (as above). Next, cells were centrifuged at 14,000 $\mathrm{g}$ for 15 minutes at $4^{\circ} \mathrm{C}$ and the soluble protein lysate was collected and quantified by BCA protein assay. Approximately $15 \mu \mathrm{g}$ of protein was resolved using 4\%-20\% gradient gel, transferred to PVDF, and probed for tau and SSAT (as described above) by Western blot analysis.
Tubulin polymerization assay. Tubulin assay was conducted using the manufacturer's protocol (Cytoskeleton, Inc., BK006P) with slight modifications. Treatments consisted of polyamines (putrescine, spermidine, spermine, acetylputrescine, acetylspermine) (Sigma-Aldrich, P5780, S4139, S4264, A8784, and O1467), $N^{1}$-acetylspermidine spermine (Cayman Chemical, 9001535). Treatments were added to a 96-well plate, which was positioned on top of an ice bath before adding tubulin. Tubulin was reconstituted and placed on ice for 2 minutes, and then added to the tubulin reaction mix. The tubulin reaction mix was then added to each well using a multichannel pipette. The plate was loaded into a BioTek Synergy H1 plate reader for 24-, 48-, 72-, 98-, and 120-minute readings at $37^{\circ} \mathrm{C}$. Each compound was assessed in triplicate wells with 3 independent tubulin preparations.

Tau-mediated tubulin morphology. Live-cell microscopy of HEK293T (ATCC, CRL-11268) cells coexpressing GFP-tubulin was performed as described previously (41) with slight modifications. Briefly, cells were transfected with GFP-tubulin constructs, and then 4 hours later treated with vehicle or $0.5 \mathrm{mM}$ or $1 \mathrm{mM}$ spermine for 48 hours. A minimum of $15-20$ cells/condition were imaged live in phenol red-free complete medium using an Olympus FV1000 MPE multiphoton laser scanning microscope. Confocal $Z$-stack images (1 $\mu \mathrm{m}$ slices) were captured using a $\times 10$ or $\times 60$ objective. Images were processed for tubulin morphology as described previously (41).

Cosedimentation assay. C3tau HeLa cells were cultured using OPTIMEM media containing $10 \%$ FBS, 100 units $/ \mathrm{mL}$ penicillin, $100 \mu \mathrm{g} / \mathrm{mL}$ streptomycin, amphotericin $\mathrm{B}$, and $0.3 \mu \mathrm{g} / \mathrm{mL} \mathrm{G}-418$. The cells were grown to $100 \%$ confluence and then washed with microtubule-stabilizing buffer (MSB) (85 mM Pipes, pH 6.93, 1 mM EGTA, $1 \mathrm{mM} \mathrm{MgCl}_{2}, 2 \mathrm{M}$ glycerol), which was kept at $37^{\circ} \mathrm{C}$ before adding to cells. Fresh MSB was 
then added to the dish while cells were removed, using a cell scraper at $37^{\circ} \mathrm{C}$ and a thermo-bead heating bath. Cells were removed, and then centrifuged at $450 \mathrm{~g}$ for 10 minutes to remove the buffer. The cell pellet was then lysed using polymerizing buffer (40 mM MES-KOH, pH 6.9, $1 \mathrm{mM}$ GTP, 1 mM DTT, 4 mM MgCl${ }_{2}$; $\%$ protease inhibitor, Sigma-Aldrich, P8340-5ML; 1\% PMSF, Sigma-Aldrich, 93482; 1\% phosphatase inhibitor cocktail 2, Sigma-Aldrich, P5726-1ML; 1\% phosphatase inhibitor cocktail 3, Sigma-Aldrich, P0044-1ML; MES-KOH) with 0.4\% Triton $\mathrm{X}-100$, which was kept at $37^{\circ} \mathrm{C}$. Cells were sonicated with 5 pulses and homogenates were centrifuged at $25,000 \mathrm{~g}$ for 15 minutes at $37^{\circ} \mathrm{C}$. The supernatant was removed and stored in a $1.5 \mathrm{~mL}$ Eppendorf tube. The pellets were resuspended in MES-KOH, incubated for 10 minutes at $4^{\circ} \mathrm{C}$, placed on ice, and separated into 4 tubes, which consisted of 4 groups: control, $0.5 \mathrm{mM}$ spermine, $1 \mathrm{mM}$ spermine, and $3 \mathrm{mM}$ spermine. The samples were incubated for 30 minutes with the appropriate polyamine at $37^{\circ} \mathrm{C}$, and then centrifuged at $25,000 \mathrm{~g}$ for 15 minutes. The supernatant was removed for Western blot analyses. The heavy pellet was resolubilized in $30 \mu \mathrm{L}$ of MES-KOH and analyzed by Western blot. A graphical representation of the assay is in Supplemental Figure 1.

ThT assay. Recombinant 4RON WT tau was purified as described previously (42). For the ThT assay, $30 \mu \mathrm{M}$ tau was combined with 10 $\mu \mathrm{M}$ ThT (Sigma-Aldrich) in $10 \mathrm{mM}$ sodium phosphate, $\mathrm{pH} 7.4$ buffer. Putrescine, spermidine, spermine, acetylputrescine, acetylspermine, $N^{1}$-acetylspermidine spermine, or vehicle control were added to black, clear-bottom 96-well plates. Aggregation was initiated with $4 \mu \mathrm{M}$ heparin, less than $2 \%$ of total well volume, and samples were incubated in a BioTek Synergy H1 plate reader for 0-24 hours and read at excitation wavelength $444 \mathrm{~nm}$ and emission wavelength $482 \mathrm{~nm}$. Each compound was assessed in triplicate wells with 3 independent tau preparations.

Split GFP-tau oligomerization assay. N2a-ssGT cells described previously (24) were used for oligomerization assays. Cells were cultured using DMEM (Gibco, 31053028) as basal medium and supplemented with $10 \%$ FBS, 1\% (v/v) GlutaMAX (2 mM), penicillin (100 IU/mL), streptomycin $(100 \mu \mathrm{g} / \mathrm{mL})$, and sodium pyruvate $(1 \mathrm{mM})$. Cells were maintained at $37^{\circ} \mathrm{C}$ in a $5 \% \mathrm{CO}_{2}$ humidified incubator. Cells were plated evenly on Costar clear-bottom, black-side 96-well plates (3603, Corning) at 10,000 per well. On the following day, cells were treated with putrescine dihydrochloride, $N$-acetylputrescine hydrochloride, spermidine, $N^{1}$-acetylspermidine hydrochloride, spermine, $N^{1}$-acetylspermine trihydrochloride (Sigma-Aldrich) at a final concentration of $30 \mu \mathrm{M}$ supplemented with 1 $\mathrm{mM}$ aminoguanidine hydrochloride (Sigma-Aldrich) to inhibit bovine amine oxidase. After 72 hours of treatment, cells were read and imaged for GFP fluorescence using a BioTek Cytation 3 reader, and then immediately harvested as a single-cell suspension medium for detecting GFP percentage by an Accuri C6 flow cytometer (BD Biosciences).

Polyamine treatment in biosensor cells. Tau RD P301S FRET biosensor cells (ATCC, CRL-3275) were cultured in DMEM media containing $10 \%$ FBS, 100 units $/ \mathrm{mL}$ penicillin, and $100 \mu \mathrm{g} / \mathrm{mL}$ streptomycin. Tau RD biosensor cells were grown to $90 \%$ confluence and reseeded in 96-well clear-bottom plates. Spermidine, spermine, acetylspermidine, and acetylspermine $(0-300 \mu \mathrm{M})$ along with $1 \mathrm{mM}$ aminoguanidine (Sigma-Aldrich) were added to Tau RD biosensor cells in serum-free media for 2 hours. Ten nanomolar (final concentration) of active human recombinant tau441 (2N4R) P301S mutant tau (tau PFFs) (StressMarq, SPR-329) complexed with Lipofectamine 2000 (1:2.5 PFF/Lipofectamine 2000) in serum-free media was added for an additional 2 hours. Tau RD biosensor cells were allowed to incubate for an additional 48 hours with polyamines and PFFs and imaged at $\times 4$ magnification using the Citation 3 Imager. Additional wells $(n=6$ wells) were harvested and collected from each treatment and subjected to flow cytometry to measure fluorescence aggregates within cells. Experiments were replicated 3 independent times.

Statistics. All statistical analyses were performed using SPSS version 25. Statistical outliers were defined as falling more than 2 standard deviations from the mean. Data are represented by mean \pm SEM scatter with bar graphs or by box-and-whisker plot displaying the minimum, first quartile, median, third quartile, and maximum. The box represents the first to third quartile, the line in the middle of the box is the median, and the whiskers span from each quartile to the minimum or maximum. A $P$ value less than 0.05 was considered significant.

A 1-way ANOVA followed by Tukey's honestly significant difference (HSD) post hoc analyses was performed for Figures 2, 15, and 16. A 1-way ANOVA followed by post hoc comparison using Dunnett's 2 -sided correction was performed for Figures 11, 12, and 14. A $2 \times 2$ factorial ANOVA followed by pairwise comparison using Sidak's posttest correction was performed for Figures 3-9. AUC was calculated using GraphPad Prism, and data are represented as averages of triplicate wells for tubulin polymerization assays and ThT assays.

For human AD Western blot analyses, $n=2$ unaffected control was removed because of Braak Stage 3 categorization and as a statistical outlier, and $n=1\left(\mathrm{AD} / \mathrm{AT}^{+}\right)$was removed from Tau 5 and AZIN2 analyses as a statistical outlier. The remaining numbers of samples $(n)$ were the following: Tau 5 (40-64 kDa) unaffected control $n=9$ (4 male, 5 female) $\left(\mathrm{AD} / \mathrm{AT}^{+}\right) n=9$ ( 3 male, 6 female), (AD/AT8 $) n=5$ (4 male, 1 female); Tau5 (100-140 kDa) unaffected control $n=9$ (4 male, 5 female), (AD/AT8 $\left.{ }^{+}\right) n=9$ ( 4 male, 5 female), (AD/AT8 $) n=5$ ( 4 male, 1 female); (AT8) unaffected controls $n=9$ ( 4 male, 5 female), (AD/AT8 ${ }^{+}$) $n=10$ (4 male, 6 female), (AD/AT8-) $n=5$ (4 male, 1 female); AZIN2 controls $n=9$ ( 4 male, 5 female), (AD/AT $\left.8^{+}\right) n=9$ ( 4 male, 5 female), $\left(\mathrm{AD} / \mathrm{AT}^{-}\right) n=5$ ( 4 male, 1 female).

A total of $n=3$ ( 1 male, 2 female) from nTg AAV9-AZIN2 group and $n=2$ ( 2 female) from PS19 AAV9-AZIN2 group viral expression outliers were removed from behavioral analyses and polyamine quantification for insufficient cortical viral expression. For the HA stain and behavioral testing, after the removal of viral outliers, group sizes were as follows: nTg AAV9-EC $n=11$ (7 male, 4 female); nTg AAV9-AZIN2: $n=6$ ( 5 male, 1 female); PS19-AAV9 EC: $n=12$ ( 6 male, 6 female); PS19 AAV9-AZIN2: $n=9$ (7 male, 2 female). Additionally, $n=1$ (female) mouse from the PS19 AAV9-AZIN2 group did not complete behavioral testing because of hind-limb paralysis; therefore, the group size for $\mathrm{Y}$ maze and inhibitory avoidance testing was $n=8$. For the HT7, AT8, and IBA1 stains, group sizes were nTg AAV9-EC: $n=7$ ( 4 male, 3 female); nTg AAV9-AZIN2: $n=3$ ( 2 male, 1 female); PS19 AAV9-EC: $n=7$ (2 male, 5 female); PS19 AAV9-AZIN2: $n=8$ ( 7 male, 1 female). For polyamine quantification, group sizes were: nTg AAV9-EC: $n=8$ (4 male, 4 female); nTg AAV9-AZIN2: $n=5$ (5 male); PS19 AAV9-EC: $n=8$ (5 male, 3 female); PS19 AAV9-AZIN2: $n=6$ ( 4 male, 2 female). A total of $n=3$ ( 2 male, 1 female) samples fell beyond the upper limit of quantification for putrescine and were removed from the PS19 AAV9-AZIN2 group. For hippocampal Western blot analyses, distributions were $\mathrm{nTg}$ AAV9-EC: $n=6$ (4 male, 2 female); nTg AAV9-AZIN2: $n=6$ (4 male, 2 female); PS19 AAV9-EC: $n=6$ (4 male, 2 female); PS19 AAV9-AZIN2: $n=6$ ( 3 male, 3 female). For hippocampal dot-blot analyses, group distributions were nTg AAV9-EC: $n=8$ ( 5 male, 3 female); nTg AAV9-AZ- 
IN2: $n=6$ ( 5 male, 1 female); PS19 AAV9-EC: $n=8$ ( 3 male, 5 female); PS19 AAV9-AZIN2: $n=6$ ( 5 male, 1 female).

Study approval. All mouse care and experimental procedures were compliant with animal experimentation guidelines and were approved by the IACUC at the University of South Florida.

\section{Author contributions}

LASB designed studies, conducted experiments, acquired data, and wrote the manuscript. AK, DSP, KR, C Ma, JBH, C Michalski, JDB, $\mathrm{SNF}$, and KRN conducted experiments, acquired data, and analyzed data. CAD provided reagents. JJG edited the manuscript. MLBS provided reagents and assisted in interpretation. HL and JITC conducted experiments, acquired data, and analyzed data on human tissue. LJB and ALD conducted experiments, acquired data, and analyzed and interpreted data on recombinant protein experiments. MF provided tissue and analysis for Alzheimer's disease hippocampal RNA. EA provided statistical analysis expertise for all data. DCL designed research studies, analyzed data, and wrote the manuscript.

\section{Acknowledgments}

We would like to thank Jun Tan and Huayan Hou (Silver Child Development Center, Morsani College of Medicine, University of
South Florida) for providing the use of instrumentation. Human brain samples from the NIH NeuroBioBank were obtained from the University of Maryland, University of Miami, and Mt. Sinai brain repositories. The graphical abstract and supplemental figure were created with BioRender.com. This work was supported by grants from the Alzheimer's Association (MNIRGD-12-242665 to DCL), Brightfocus Foundation (A20155045 to DCL), CurePSP, Inc. (520-14 to DCL), Florida Department of Health (5AZ11 to DCL), and NIH R01AG054559 (to DCL).

Address correspondence to: Daniel C. Lee, Sanders-Brown Center on Aging, Department of Neuroscience, The University of Kentucky, 800 S. Limestone St., Lexington, Kentucky 40536, USA. Phone:1.859.323.6506; Email: dan.lee@uky.edu.

LASB's present address is: Neuroscience Institute, Department of Neuroscience and Physiology, New York University School of Medicine, New York, New York, 10016, USA.

SNF's present address is: Ripple Effect Communications, Inc., Congressionally Directed Medical Research Programs (CDMRP), Fort Detrick, Maryland 21702-5024, USA.
1. Spillantini MG, Goedert M. Tau pathology and neurodegeneration. Lancet Neurol. 2013;12(6):609-622.

2. Liu $\mathrm{P}$, et al. Altered arginine metabolism in Alzheimer's disease brains. Neurobiol Aging. 2014;35(9):1992-2003.

3. Inoue K, et al. Metabolic profiling of Alzheimer's disease brains. Sci Rep. 2013;3:2364.

4. Sigrist SJ, et al. Spermidine-triggered autophagy ameliorates memory during aging. Autophagy. 2014;10(1):178-179.

5. Signor C, et al. Spermidine improves fear memory persistence. Eur J Pharmacol. 2014;730:72-76.

6. Gupta VK, et al. Restoring polyamines protects from age-induced memory impairment in an autophagy-dependent manner. Nat Neurosci. 2013;16(10):1453-1460.

7. Guerra GP, et al. Spermidine-induced improvement of memory involves a cross-talk between protein kinases $\mathrm{C}$ and A. J Neurochem. 2012;122(2):363-373.

8. Pegg AE. Regulation of ornithine decarboxylase. J Biol Chem. 2006;281(21):14529-14532.

9. Mangold U. The antizyme family: polyamines and beyond. IUBMB Life. 2005;57(10):671-676.

10. Gilad GM, Gilad VH. Overview of the brain polyamine-stress-response: regulation, development, and modulation by lithium and role in cell survival. Cell Mol Neurobiol. 2003;23(4-5):637-649.

11. Park MH, Igarashi K. Polyamines and their metabolites as diagnostic markers of human diseases. Biomol Ther (Seoul). 2013;21(1):1-9.

12. Tabor CW, Tabor H. Polyamines. Annu Rev Biochem. 1984;53:749-790.

13. Zahedi K, et al. Polyamine catabolism is enhanced after traumatic brain injury. J Neurotrauma. 2010;27(3):515-525.

14. Pegg AE, et al. Current status of the polyamine research field. Methods Mol Biol. 2011;720:3-35.

15. Pan X, et al. Alzheimer's disease-like pathology has transient effects on the brain and blood metabolome. Neurobiol Aging. 2016;38:151-163.

16. Wang Y, et al. Characterization of the interaction between the transcription factors human polyamine modulated factor (PMF-1) and NF-E2-related factor 2 (Nrf-2) in the transcriptional regulation of the spermidine/spermine N1-acetyltransferase (SSAT) gene. Biochem J. 2001;355(pt 1):45-49.

17. Hiasa M, et al. Identification of a mammalian vesicular polyamine transporter. Sci Rep. 2014;4:6836

18. Nishimura K, et al. Decrease in polyamines with aging and their ingestion from food and drink. $J$ Biochem. 2006;139(1):81-90.

19. Jasper TW, et al. Polyamines in the developing mouse brain. Dev Neurosci. 1982;5(2-3):233-242.

20. Makitie LT, et al. Brain neurons express ornithine decarboxylase-activating antizyme inhibitor 2 with accumulation in Alzheimer's disease. Brain Pathol. 2010;20(3):571-580.

21. Graham SF, et al. Untargeted metabolomic analysis of human plasma indicates differentially affected polyamine and L-arginine metabolism in mild cognitive impairment subjects converting to Alzheimer's disease. PLoS One. 2015;10(3):e0119452.

22. Yi J, et al. L-arginine and Alzheimer's disease. Int J Clin Exp Pathol. 2009;2(3):211-238.

23. Morrison LD, et al. Ornithine decarboxylase in human brain: influence of aging, regional distribution, and Alzheimer's disease. J Neurochem. 1998;71(1):288-294.

24. Sandusky-Beltran LA, et al. Spermidine/spermine- $\mathrm{N}^{1}$-acetyltransferase ablation impacts tauopathy-induced polyamine stress response. Alzheimers Res Ther. 2019;11(1):58.

25. Guerra GP, et al. Modulation of learning and memory by natural polyamines. Pharmacol Res. 2016;112:99-118.
26. Limon A, et al. Targets of polyamine dysregulation in major depression and suicide: activity-dependent feedback, excitability, and neurotransmission. Neurosci Biobehav Rev. 2016;66:80-91.

27. Chen GG, et al. Evidence of altered polyamine concentrations in cerebral cortex of suicide completers. Neuropsychopharmacology. 2010;35(7):1477-1484.

28. Fiori LM, Turecki G. Genetic and epigenetic influences on expression of spermine synthase and spermine oxidase in suicide completers. Int $J$ Neuropsychopharmacol. 2010;13(6):725-736.

29. Fiori LM, et al. Association of polyaminergic loci with anxiety, mood disorders, and attempted suicide. PLoS One. 2010;5(11):e15146.

30. Takeuchi H, et al. P301S mutant human tau transgenic mice manifest early symptoms of human tauopathies with dementia and altered sensorimotor gating. PLoS One. 2011;6(6):e21050.

31. Lasagna-Reeves CA, et al. Reduction of Nuak1 decreases tau and reverses phenotypes in a tauopathy mouse model. Neuron. 2016;92(2):407-418.

32. Iba M, et al. Synthetic Tau fibrils mediate transmission of neurofibrillary tangles in a transgenic mouse model of Alzheimer's-like tauopathy. J Neurosci. 2013;33(3):1024-1037.

33. Vemula $P$, et al. Altered brain arginine metabolism in a mouse model of tauopathy. Amino Acids. 2019;51(3):513-528.

34. Lefevre J, et al. The $\mathrm{C}$ terminus of tubulin, a versatile partner for cationic molecules: binding of tau, polyamines, and calcium. J Biol Chem. 2011;286(4):3065-3078.

35. LaFerla FM. Calcium dyshomeostasis and intracellular signalling in Alzheimer's disease. Nat Rev Neurosci. 2002;3(11):862-872.

36. Michalski B, et al. Brain-derived neurotrophic factor and TrkB expression in the "oldest-old," the 90+ Study: correlation with cognitive status 
and levels of soluble amyloid-beta. Neurobio Aging. 2015;36(12):3130-3139.

37. Yoshiyama Y, et al. Synapse loss and microglial activation precede tangles in a P301S tauopathy mouse model. Neuron. 2007;53(3):337-351.

38. Carty N, et al. Convection-enhanced delivery and systemic mannitol increase gene product distribution of AAV vectors 5, 8, and 9 and increase gene product in the adult mouse brain. J Neurosci
Methods. 2010;194(1):144-153.

39. Carty N, et al. Intracranial injection of AAV expressing NEP but not IDE reduces amyloid pathology in APP+PS1 transgenic mice. PLoS One. 2013;8(3):e59626.

40. Hunt JB Jr, et al. Sustained arginase 1 expression modulates pathological tau deposits in a mouse model of tauopathy. J Neurosci. 2015;35(44):14842-14860.
41. Fontaine SN, et al. The active Hsc70/tau complex can be exploited to enhance tau turnover without damaging microtubule dynamics. Hum Mol Genet. 2015;24(14):3971-3981.

42. Fontaine $\mathrm{SN}$, et al. Isoform-selective genetic inhibition of constitutive cytosolic Hsp70 activity promotes client tau degradation using an altered co-chaperone complement. J Biol Chem. 2015;290(21):13115-13127. 